\title{
Length two extensions of modules for the Witt algebra
}

\author{
Kathlyn Dykes \\ A thesis submitted to \\ the Faculty of Graduate and Postdoctoral Affairs \\ in partial fulfillment of the requirements \\ for the degree of Master of Science \\ in Mathematics \\ Carleton University \\ Ottawa, Ontario
}

(C)2015, Kathlyn Dykes 


\section{Abstract}

In this thesis, we analyse length two extensions of tensor modules for the Witt algebra. In 1992, a classification of these modules was found by Martin and Piard in [14], though no explicit form of the extensions were given. In this thesis, we establish an explicit classification of these modules using a different approach. As we will show, each module extension is classified by a 1-cocycle from the cohomology of the Witt algbera with coefficients in the module of the space of homomorphisms between the two tensor modules of interest. To use this, we first extended our module to a module that has a compatible action of the commutative algebra of Laurent polynomials in one variable. In this setting, we are able to determine the possible structure of a 1-cocycle and from here, we will be able to directly compute all possible 1-cocycles. 


\section{Contents}

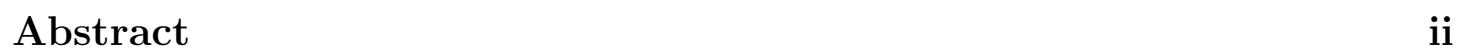

$\begin{array}{lll}1 & \text { Introduction } & 1\end{array}$

\begin{tabular}{|lll}
\hline 2 & Background & 4
\end{tabular}

2.1 The Witt algebra and its irreducible modules . . . . . . . . . . . . . 4

2.2 The $A$-cover of a $W_{1}$-module $\ldots \ldots \ldots \ldots$

2.3 Cohomology of Lie algebras _ . . . . . . . . . . . . . . . . . . . 10

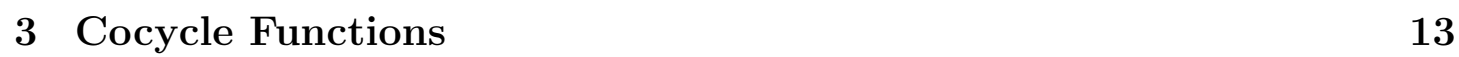

3.1 Parameters of the module extension . . . . . . . . . . . . . . . . . 13

3.2 A new module extension . . . . . . . . . . . . . . . . . . . . . . . . 18

$3.3 \quad$ Submodules of $\operatorname{Hom}(A, T(\alpha, \gamma))$ with finite dimensional weight spaces 23

$3.4 \quad$ Finding an appropriate basis for $M \ldots \ldots \ldots$

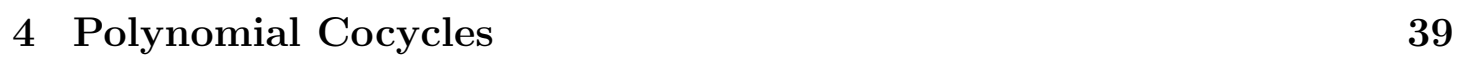

4.1 General results for polynomial cocycles . . . . . . . . . . . . . . 39

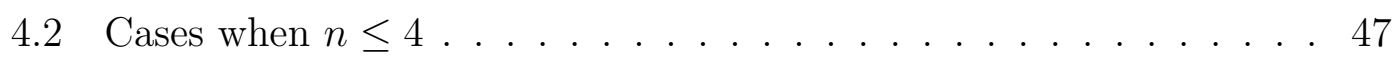

$4.2 .1 \quad$ Cocycles of degree $1 \ldots \ldots . \ldots . \ldots 47$

$4.2 .2 \quad$ Cocycles of degree 2

$4.2 .3 \quad$ Cocycles of degree $3 \ldots \ldots \ldots$ 
$4.2 .4 \quad$ Cocycles of degree $4 \ldots \ldots \ldots \ldots$

4.3 Cases when $n \geq 5$. . . . . . . . . . . . . . . . . . 54

$4.3 .1 \quad$ Cocycles of degree 5 . . . . . . . . . . . . . . . . . . 59

$4.3 .2 \quad$ Cocycles of degree 6 . . . . . . . . . . . . . . . . 59

$4.3 .3 \quad$ Cocycles of degree 7 . . . . . . . . . . . . . . . . 60

4.3 .4 Cocycles of degree greater than or equal to 8 . . . . . . . . 61

$4.4 \quad$ Dual Modules . . . . . . . . . . . . . . . . . . . . . . . . . . . . . 62

$\begin{array}{lll}5 & \text { Delta Cocycles } & 65\end{array}$

5.1 Conditions for delta functions $\ldots \ldots$. . . . . . . . . . . . 66

\begin{tabular}{lll}
\hline 6 & Special case of $\beta=1$ & 70
\end{tabular}

6.1 Conditions on non-polynomial cocycles . . . . . . . . . . . . . 70

6.2 Cocycles with a factor of $m^{-1} \ldots \ldots \ldots 73$

6.3 Delta cocycles . . . . . . . . . . . . . . . . . . . . 75

\begin{tabular}{lll}
\hline 7 & Conclusion & 79
\end{tabular}

\begin{tabular}{lr}
\hline References & 85
\end{tabular} 


\section{List of Tables}

7.1 Polynomial cocycles in $\widetilde{M}_{N} \ldots \ldots \ldots \ldots \ldots$

7.2 Polynomial cocycles in $M \ldots \ldots \ldots$. . . . . . . 81

7.3 Non-polynomial cocycles $\ldots \ldots \ldots$. . . . . . . . . . 82 


\section{Chapter 1}

\section{Introduction}

This thesis is concerned with classifying all length two extensions of tensor modules of the Witt algebra, $W_{1}$. This is a question that has been studied before by Martin and Piard in their 1992 paper [14]. The classification is given here explicitly, which is something that has been lacking in previous works. By comparison, our results are similar to the classification of the cohomology of the Lie algebra of vector fields on a line given by Feigin and Fuks in their 1982 paper [8].

The Witt algebra can be described in a few different ways. It is the Lie algebra of derivations of Laurent polynomials in one variable. It is also the complexification of the Lie algebra of real vector fields on the circle, or can be described as the Lie algebra of the group of (orientation preserving) diffeomorphisms of the circle[[12].

In conformal field theory, the Witt algebra is described as the Lie algebra of the conformal group of the complex plane, where the conformal group is the group of transformations that preserve the angle between any two vectors. In quantum field theory, the Virasoro algebra, the central extension of the Witt algebra, is considered instead [10].

The importance of the Witt algebra is strongly linked to its central exten- 
sion. The Virasoro algebra has many important roles in the area of mathematical physics. From conformal field theory to soliton theory to string theory, representations of the Virasoro algebra are studied extensively [12]. In this paper, we will find module extensions of the Witt algebra that admit delta-cocycles. These cocycles can be used to construct Lie algebras that contain the Virasoro algebra as a subalgebra. One of these algebras, the $W(2,2)$ algebra, can be built from the semidirect product of Virasoro algebra and its adjoint representation. This algebra has a very different representation theory than that of the Virasoro algebra; the highest weight modules for $W(2,2)$ admit vertex operator realizations [17]. Similarly, we can define the twisted Heisenberg-Virasoro algebra which has applications in the study of the representations of the toroidal Lie algebra [18],

In his 1992 paper [15], Mathieu gave a complete classification of irreducible Virasoro modules with finite dimensional weight spaces. Consequently, as any Witt algebra module is a Virasoro module where the central element acts trivially, his results are also applicable to Witt algebra modules. As was proved in[15], every irreducible $W_{1}$-module with finite-dimensional weight spaces is either a highest weight modules, a lowest weight module, a tensor module or quotient of a tensor module. In this thesis, we consider only tensor modules of the Witt algebra.

The problem of classifying length two extensions of modules can be solved with the help of cohomology. We will make use of the objects of cocycles and coboundaries, which will in turn completely determine the module extensions.

In this paper, the method used to obtain the classification of these module extensions $M$, begins with moving up to the $A$-cover $\widehat{M}$. Though it turns out that this space is too big, the idea is to obtain a classification in the context of $A W_{1}$-modules, which is a simpler problem computationally than in the $W_{1}$-module setting. We obtain a surjection from the space where the classification is easily 
calculated back to the original module, which will give a classification of these extensions.

These results are important in two ways. First, no explicit classification of these modules had been previously presented. Another aspect of these results is the method used to obtain this classification. Instead of working in the modules of the Witt algebra, we lift the module into the context of $A W_{1}$-modules, which are modules of the Witt algebra which have a compatible action with the commutative algebra of Laurent polynomials in one variable. In fact, we can extend this definition to define an $A_{n} W_{n}$-module[5]. The methods used in this work will extend to the case of $W_{n}$, giving us somewhere to start in the classification of length two extensions of $W_{n}$-modules.

This thesis is organized as follows: In the Chapter 2, we will introduce the main areas of study that will be used throughout the remaining chapters. This will include a description of the Witt algebra and the module extensions we are interested in. We will introduce the notion of an $A$-cover of a module of the Witt algebra and present enough cohomology of Lie algebras to serve our purposes. In Chapter 3 , the structure of module extensions is shown to be completely determined by 1-cocycles. These functions are studied in a general setting and the conditions necessary for cocycles to be polynomial are found. In Chapter 4, we classify all polynomial cocycles. In Chapter 5, we classify all delta function cocycles. In Chapter 6 , we look at the special case of $\beta=1$, where $\beta$ is one of the parameters of the module extension. Finally, we end with a summary of all the results. 


\section{Chapter 2}

\section{Background}

In this section, we will discuss three topics that are used in the body of this thesis. Lie algebra modules are the objects we are concerning ourselves with, in particular Witt algebra modules. We will introduce the tensor modules of the Witt algebra and then we will introduce the length two module extensions that will be investigated. We will briefly discuss the notion of an A-cover of a $W_{1}$-module, which will be a crucial tool in our investigation of the structure of these modules. At the end of this chapter, we briefly discuss enough cohomology theory to be of use. We will also introduce the notations that will be used throughout this thesis.

\subsection{The Witt algebra and its irreducible modules}

In general, we can define a commutative associative algebra $A_{n}$ for $n \in \mathbb{N}$ by

$$
A_{n}=\mathbb{C}\left[t_{1}^{ \pm 1}, t_{2}^{ \pm 1}, \ldots, t_{n}^{ \pm 1}\right]
$$


which is the algebra of Laurent polynomials in $n$ variables with the regular polynomial multiplication.

A derivation of an algebra $L$ is defined to be a map $d: L \rightarrow L$ such that $d(x y)=d(x) y+x d(y)$ for all $x, y \in L$. Notice this is very similar to the product rule on derivatives of functions. The most basic example of a derivation of a Lie algebra is the adjoint representation defined by ad $x: L \rightarrow L$ where $\operatorname{ad} x(y)=[x, y]$ for all $y \in L$.

If we look at the set of all derivations of $A_{n}$, we obtain a Lie algebra, denoted by $W_{n}$. By introducing $d_{i}=t_{i} \frac{\partial}{\partial t_{i}}$ for $i=1, \ldots, n$, then this Lie algebra is spanned by the vectors $\left\{t_{1}^{k_{1}} \ldots t_{n}^{k_{n}} d_{i} \mid k_{1}, \ldots, k_{n} \in \mathbb{Z}, i=1, \ldots, n\right\}[5]$.

The Lie algebra we are interested in is the Witt algebra and is denoted by $W_{1}$. As the notation indicates, the Witt algebra is the case of $W_{n}$ when $n=1$. By simplifying the notations, we denote $A_{1}$ by $A=\mathbb{C}\left[t^{ \pm 1}\right]$ so that the Witt algebra is the Lie algebra of derivations of $A$. In this case, we only have $d_{1}=t \frac{d}{d t}$ so that $W_{1}$ is spanned by the basis vectors $\left\{e_{k}=t^{k+1} \frac{d}{d t} \mid k \in \mathbb{Z}\right\}$.

The bracket of $W_{1}$ is given by

$$
e_{k} \cdot e_{s}-e_{s} \cdot e_{k}=\left[e_{k}, e_{s}\right]=(s-k) e_{k+s}
$$

where the product on the left is the associative product $t^{k+1} \frac{d}{d t} t^{s+1} \frac{d}{d t}$ of derivations.

As discussed in the introduction, the main interest in the Witt algebra is its central extension. The Virasoro algebra is defined by $W_{1} \oplus \mathbb{C} c$, where $c$ commutes with everything and is called the central element. By using the notation that $\delta_{j+k, 0}$ denotes the function

$$
\delta_{j+k, 0}=\left\{\begin{array}{cc}
1, & j=-k \\
0, & j \neq-k
\end{array}\right.
$$


we can write the bracket of the Virasoro algebra as

$$
\begin{aligned}
{\left[e_{k}, e_{s}\right] } & =(s-k) e_{s+k}+\delta_{s+k, 0}\left(\frac{k^{3}-k}{12}\right) c \\
{\left[c, e_{k}\right] } & =0
\end{aligned}
$$

We will now introduce the types of modules that we will concern ourselves with. In particular, we will only work with modules that have finite dimensional weight spaces. We first need to introduce the notion of weight spaces.

The normalizer of a subalgebra $K$ of a Lie algebra $L$ is the subalgebra $N_{L}(K)$ defined by

$$
N_{L}(K)=\{x \in L \mid \forall k \in K,[x, k] \in K\}
$$

The descending central series of a Lie algebra is defined by the subalgebras $L_{n}$ where $L_{0}=L, L_{n}=\left[L, L_{n-1}\right]$. If there exists some $k \in \mathbb{Z}$ such that $L_{k}=\{0\}$, then this Lie algebra is called nilpotent.

Definition 2.1.1. A Cartan subalgebra $H$ of a Lie algebra $L$ is a nilpotent subalgebra satisfying $N_{L}(H)=H$.

We consider Cartan subalgebras with an additional property of being MAD (maximal abelian diagonalizable) subalgebras. These subalgebras are abelian and act diagonally on $L$, and are not strictly contained in any subalgebra with these two properties. In $W_{1}, \mathbb{C} e_{0}$ is the MAD subalgebra [5]

Definition 2.1.2. A module $V$ of a Lie algebra $L$ with Cartan subalgebra $H$ is a weight module if there exist weights $\lambda \in H^{*}$ such that $V=\bigoplus_{\lambda} V_{\lambda}$, where

$$
V_{\lambda}=\{v \in V \mid \forall h \in H, h . v=\lambda(h) v\}
$$

In $W_{1}$, we can simplify this definition of a weight module using that $H=\mathbb{C} e_{0}$. 
Definition 2.1.3. A $W_{1}$-module $V$ is a weight module if there exist weights $\lambda \in \mathbb{C}$ such that $V=\oplus_{\lambda} V_{\lambda}$ where the weight space of weight $\lambda$ is given by

$$
V_{\lambda}=\left\{v \in V \mid e_{0} \cdot v=\lambda v\right\}
$$

Definition 2.1.4. A weight module is called cuspidal if the dimensions of all weight spaces are bounded by a common constant.

In the $W_{n}$ case, we can consider tensor modules which are parameterized by a complex vector $\gamma \in \mathbb{C}^{n}$ and a finite-dimensional $g l_{n}$-module as given in [5], In the 1-dimensional case, $g l_{1}=\mathbb{C}$ so that tensor modules are parameterized by two constants, $\gamma, \alpha \in \mathbb{C}$.

The structure of these tensor $W_{1}$-modules, denoted by $T(\alpha, \gamma)$, are given by

$$
T(\alpha, \gamma)=\bigoplus_{m \in \gamma+\mathbb{Z}} \mathbb{C} v_{m}
$$

with $W_{1}$ action

$$
e_{k} \cdot v_{m}=(m+\alpha k) v_{m+k}
$$

Notice that $e_{0} v_{k}=k v_{k}$, so these modules have 1 -dimensional weight spaces $\mathbb{C} v_{k}$ with weights $k \in \gamma+\mathbb{Z}$. As the dimension of all weight spaces are bounded by 1 , tensor $W_{1}$-modules are in fact cuspidal modules.

One thing to remark before proceeding further is that $T(\gamma, \alpha) \cong T\left(\gamma^{\prime}, \alpha\right)$ if $\gamma-\gamma^{\prime} \in \mathbb{Z}$, as stated in Remark 1.1 of [12]. Therefore, we will always assume that $0 \leq \operatorname{Re}(\gamma)<1$. Consequently, we will treat the cases of $\gamma \in \mathbb{Z}$ and $\gamma=0$ as interchangeable.

These tensor modules are irreducible except in two special cases: $\alpha=0, \gamma \in \mathbb{Z}$ and $\alpha=1, \gamma \in \mathbb{Z}$ by Proposition 1.1 of [12]. For convenience, we will denote by 
$D(0,0)=\operatorname{span}\left\{v_{0}\right\}, T^{\circ}(1,0)=\operatorname{span}\left\{v_{m} \mid m \in \mathbb{Z}, m \neq 0\right\} . D(0,0)$ is a submodule of $T(0,0)$ and $T^{\circ}(1,0)$ is a submodule of $T(1,0)$, as shown below.

$$
\begin{array}{r}
e_{k} v_{0}=0, \forall k \in \mathbb{Z} \text { in } T(0,0) \\
e_{k} v_{-k}=0, \forall k \in \mathbb{Z} \text { in } T(1,0)
\end{array}
$$

In this thesis, we will concern ourselves with weight $W_{1}$-modules $M$ which possess the short exact sequence:

$$
0 \rightarrow T(\alpha, \gamma) \rightarrow M \rightarrow T\left(\beta, \gamma^{\prime}\right) \rightarrow 0
$$

In this notation, each arrow represents a homomorphism so that $M$ has a submodule $T(\alpha, \gamma)$ with the quotient $M / T(\alpha, \gamma) \cong T\left(\beta, \gamma^{\prime}\right)$. This extension is also taken to be a weight extension, meaning that the weight space $M_{\lambda}$ is given by the exact sequence

$$
0 \rightarrow T(\alpha, \gamma)_{\lambda} \rightarrow M_{\lambda} \rightarrow T\left(\beta, \gamma^{\prime}\right)_{\lambda} \rightarrow 0
$$

One final remark about these tensor modules is that every irreducible cuspidal module of $W_{1}$ is isomorphic to either a tensor modules, $D(0,0)$ or $T^{\circ}(1,0)$, as the only cuspidal highest and lowest weight modules are trivial (Corollary III.3 of [13]). We will consider only tensor modules extensions, but the cases of $D(0,0)$ and $T^{\circ}(1,0)$ will come out of these. 


\subsection{The $A$-cover of a $W_{1}$-module}

Another tool that will be needed in this paper is the idea of an $A$-cover of a $W_{1}$-module. First, notice that $W_{1}$ is an $A$-module with the $A$-action:

$$
t^{k} e_{m}=e_{m+k}
$$

and $A$ is a $W_{1}$-module with the $W_{1}$-action:

$$
e_{k} t^{m}=m t^{m+k}
$$

This action can be extended linearly to all $f \in A$ and all $x \in W_{1}$.

If $V$ is both an $A$-module and a $W_{1}$-module, then the $A$-action and the $W_{1^{-}}$ action is compatible if

$$
(x f) v=x(f v)-f(x v), \text { for all } x \in W_{1}, f \in A, v \in V
$$

If $V$ has a compatible $A$-action and $W_{1}$ action, it is called an $A W_{1}$-module [5]

The space of $\operatorname{Hom}_{\mathbb{C}}(A, V)$ is an example of $A W_{1}$-module [5], with the actions

$$
\begin{aligned}
& (x \psi)(f)=x(\psi(f))-\psi(x(f)) \\
& (g \psi)(f)=\psi(g f), \text { for } \psi \in \operatorname{Hom}(A, V), x \in W_{1}, f, g \in A
\end{aligned}
$$

We now introduce a submodule of $\operatorname{Hom}(A, V)$ which will be of use to us throughout the next chapter.

Definition 2.2.1. If $V$ is a $W_{1}$-module, the $A$-cover $\widehat{V}$ is defined as

$$
\widehat{V}=\operatorname{span}\left\{\phi(x, u) \mid x \in W_{1}, u \in V\right\} \subset \operatorname{Hom}(A, V)
$$


where $\phi(x, u)(f)=(f x)(u)$ for all $f \in A$.

The $A$-cover is an $A W_{1}$-submodule of $\operatorname{Hom}(A, V)$. By Proposition 4.5 of [5], if $V$ is a weight module, then so is $\widehat{V}$. In fact, if $V$ is a cuspidal module, then so if $\widehat{V}$, as shown in Theorem 4.10 of [5]. The $W_{1}$-action and $A$-action on the $A$-cover is

$$
\begin{aligned}
& y \phi(x, u)=\phi([y, x], u)+\phi(x, y u) \\
& g \phi(x, u)=\phi(g x, u)
\end{aligned}
$$

for $x, y \in W_{1}, u \in V, g \in A$.

From $\widehat{V}$, we can always go back to the space $V$ via the map $\pi: \widehat{V} \rightarrow V$ where $\pi(\phi(x, u))=\phi(x, u)(1)=x . u$. Notice this map is only surjective if $W_{1} V=V$ (Proposiiton 4.5 of [5]).

\subsection{Cohomology of Lie algebras}

For this section, suppose that $L$ is a Lie algebra and $V$ is an $L$-module.

Definition 2.3.1. An $n$-dimensional cochain of $L$ with coefficients in $V$ is a skewsymmetric linear map $L^{\otimes^{n}} \rightarrow V$. The space of all cochains is denoted by $C^{n}(L, V)$.

Equivalently, $C^{n}(L, V)=\operatorname{Hom}\left(\wedge^{n} L, V\right)$ as the wedge product is skew-symmetric. These spaces $C^{n}(L, V)$ can be related to each other using the following maps:

$$
d_{n}: C^{n}(L, V) \rightarrow C^{n+1}(L, V)
$$


where, for $\varphi \in C^{n}(L, V), g_{1}, \ldots, g_{n+1} \in L$, the map $d_{n}$ is defined as

$$
\begin{aligned}
& d_{n} \varphi\left(g_{1}, \ldots, g_{n+1}\right)=\sum_{1 \leq i \leq n+1}(-1)^{i} g_{i} \varphi\left(g_{1}, \ldots, g_{i-1}, g_{i+1}, \ldots, g_{n+1}\right) \\
& +\sum_{1 \leq i<j \leq n+1}(-1)^{i+j-1} \varphi\left(\left[g_{i}, g_{j}\right], g_{1}, \ldots, g_{i-1}, g_{i+1}, \ldots, g_{j-1}, g_{j+1}, \ldots, g_{n+1}\right)
\end{aligned}
$$

For $n<0, C^{n}(L, V)=0$ and $d_{n}=0$. In fact, $d_{n+1} \circ d_{n}=0$ (page 15 of [9] ) so that the set of $C^{n}(L, V)$ together with the maps $d_{n}$ is an algebraic complex. We now have the tools to define the cohomology of the Lie algebra $L$.

Definition 2.3.2. The cohomology of $L$ with coefficients in $V$, denoted by $H^{*}(L, V)$, is the space $H^{*}(L, V)=\bigoplus_{n=0}^{\infty} H^{n}(L, V)$, where

$$
H^{n}(L, V)=\operatorname{Ker}\left(d_{n}\right) / \operatorname{Im}\left(d_{n-1}\right)
$$

An element in the kernel of the $d_{n}$ map is called an $n$-cocyle. An element in the image of the $d_{n-1}$ map is called an $n$-coboundary.

The space $\operatorname{Hom}_{\mathbb{C}}\left(T\left(\beta, \gamma^{\prime}\right), T(\alpha, \gamma)\right)$ is a $W_{1}$-module with module action given by

$$
\left(e_{k} \cdot x\right)(w)=e_{k} \cdot(x(w))-x\left(e_{k} \cdot w\right)
$$

As we are considering graded modules, we will instead look at the graded version of $\operatorname{Hom}_{\mathbb{C}}\left(T\left(\beta, \gamma^{\prime}\right), T(\alpha, \gamma)\right)$. It is the space of the set of maps $\varphi \in \operatorname{Hom}_{\mathbb{C}}\left(T\left(\beta, \gamma^{\prime}\right), T(\alpha, \gamma)\right)$ such that $\varphi\left(w_{m}\right) \in \mathbb{C} v_{m+k}$ for all $m \in \gamma^{\prime}+\mathbb{Z}$, or elements that shift the grading of the module $T(\alpha, \gamma)$ by $k$. This is still a module with $W_{1}$-action as defined above.

We will consider the cohomology of $W_{1}$ with values in the graded module $\operatorname{Hom}_{\mathbb{C}}\left(T\left(\beta, \gamma^{\prime}\right), T(\alpha, \gamma)\right)$. Again, as we are working with graded spaces, we will consider the cohomology that is compatible with the $\mathbb{Z}$-grading of this module. Thus, we only consider $n$-cochains from $\wedge^{n} L \rightarrow V$ that preserve the $\mathbb{Z}$-grading. 
Then

$$
\begin{aligned}
C^{0}\left(W_{1}, \operatorname{Hom}\left(T\left(\beta, \gamma^{\prime}\right), T(\alpha, \gamma)\right)\right) & =\operatorname{Hom}\left(\mathbb{C}, \operatorname{Hom}\left(T\left(\beta, \gamma^{\prime}\right), T(\alpha, \gamma)\right)\right) \\
& \cong \operatorname{Hom}\left(T\left(\beta, \gamma^{\prime}\right), T(\alpha, \gamma)\right) \\
C^{1}\left(W_{1}, \operatorname{Hom}\left(T\left(\beta, \gamma^{\prime}\right), T(\alpha, \gamma)\right)\right) & =\operatorname{Hom}\left(W_{1}, \operatorname{Hom}\left(T\left(\beta, \gamma^{\prime}\right), T(\alpha, \gamma)\right)\right) \\
& \cong \operatorname{Hom}\left(W_{1} \otimes T\left(\beta, \gamma^{\prime}\right), T(\alpha, \gamma)\right)
\end{aligned}
$$

1-cocycles are in the kernel of $d_{1}$ and 1-coboundaries are in the image of $d_{0}$ and $d_{1} \circ d_{0}=0$ as shown below for $\varphi \in \operatorname{Hom}\left(\mathbb{C}, \operatorname{Hom}\left(T\left(\beta, \gamma^{\prime}\right), T(\alpha, \gamma)\right)\right), g_{1}, g_{2} \in W_{1}$.

$$
\begin{aligned}
\left(d_{1} \circ d_{0}(\varphi)\right)\left(g_{1}, g_{2}\right) & =d_{1}\left(d_{0}(\varphi)\right)\left(g_{1}, g_{2}\right) \\
& =g_{2} d_{0}(\varphi)\left(g_{1}\right)-g_{1} d_{0}(\varphi)\left(g_{2}\right)+d_{0}(\varphi)\left(\left[g_{1}, g_{2}\right]\right) \\
& =-g_{2} g_{1} \varphi+g_{1} g_{2} \varphi-\left[g_{1}, g_{2}\right] \varphi \\
& =\left(\left[g_{2}, g_{1}\right]-g_{2} g_{1}+g_{1} g_{2}\right) \varphi \\
& =0
\end{aligned}
$$

These concepts will be useful in our efforts to understand and classify length two extensions of the tensor modules of the Witt algebra. 


\section{Chapter 3}

\section{Cocycle Functions}

In this section, we look at the module extension

$$
0 \rightarrow T(\alpha, \gamma) \rightarrow M \rightarrow T\left(\beta, \gamma^{\prime}\right) \rightarrow 0
$$

where $T(\alpha, \gamma)$ is spanned by vectors $v_{m}$ and $T\left(\beta, \gamma^{\prime}\right)$ is spanned by vectors $w_{m}$. In this way, $M$ is spanned by basis vectors $v_{m}$ and $w_{m}$, where these $w_{m} \in M$ are mapped to $w_{m} \in T\left(\beta, \gamma^{\prime}\right)$ under the surjection from $M$ to $T\left(\beta, \gamma^{\prime}\right)$.

We will introduce how we can use the notion of a cocycle to classify the module extensions. Then we will use the A-cover of our module $M$ to find an appropriate basis of $M$ so that the corresponding cocycles will be polynomial, except in a few special cases.

\subsection{Parameters of the module extension}

Since $T(\alpha, \gamma)$ is a $W_{1}$-submodule of $M$, then the $W_{1}$ action is, by definition,

$$
e_{k} \cdot v_{m}=(m+\alpha k) v_{m+k}, \text { for } k \in \mathbb{Z}, m \in \gamma+\mathbb{Z}
$$


The $W_{1}$-action on the $w_{m}$ basis vectors is defined as

$$
e_{k} \cdot w_{m}=(m+\beta k) w_{m+k}+\tau(k, m) v_{m+k}, \text { for } k \in \mathbb{Z}, m \in \gamma^{\prime}+\mathbb{Z}
$$

where $\tau(k, m)$ is some function in $k$ and $m$.

Here, we can see that $M$ is parameterized by two objects: the function $\tau(k, m)$ and the complex number $\gamma$. First, let us look at the relation between $\gamma$ and $\gamma^{\prime}$.

Lemma 3.1.1. If $\gamma+\mathbb{Z} \neq \gamma^{\prime}+\mathbb{Z}$, then $M=T(\alpha, \gamma) \bigoplus T\left(\beta, \gamma^{\prime}\right)$.

Proof. The $W_{1}$-action is given by

$$
e_{k} w_{m}=(m+\alpha k) w_{m+k}+\tau(k, m) v_{m+k}
$$

but since $m+k \notin \gamma+\mathbb{Z}, \tau(k, m)$ must be zero.

As long as $\gamma \neq \gamma^{\prime}$, the extension will be trivial in the sense that $\tau(k, m)$ is the zero function. Since we are interested in non-trivial extensions we will only consider the case that $\gamma+\mathbb{Z}=\gamma^{\prime}+\mathbb{Z}$. If $\gamma+\mathbb{Z}=\gamma^{\prime}+\mathbb{Z}$, then $T\left(\beta, \gamma^{\prime}\right) \cong T(\beta, \gamma)$ so that we may assume $\gamma=\gamma^{\prime}$ for the rest of this thesis.

Now, let us look at the conditions on $\tau(k, m) . M$ is a $W_{1}$-module and it follows that the $W_{1}$-action must be a module action.

$$
\begin{aligned}
{\left[e_{k}, e_{s}\right] w_{m}=} & \left(e_{k} e_{s}-e_{s} e_{k}\right) w_{m} \\
(s-k) e_{k+s} w_{m}= & (m+\beta s) e_{k} w_{m+s}+e_{k} \tau(s, m) v_{m+s} \\
& -(m+\beta s) e_{s} w_{m+k}-e_{s} \tau(k, m) v_{m+k} \\
\Longrightarrow(s-k)(m+\beta(k+s)) w_{m+k+s}= & (m+\beta s)(m+s+\beta k) w_{m+s+k} \\
& -(m+\beta k)(m+k+\beta s) w_{m+k+s} \\
\Longrightarrow(s-k) \tau(k+s, m) v_{m+s+k}= & (m+\beta s) \tau(k, m+s) v_{m+k+s}
\end{aligned}
$$




$$
\begin{aligned}
& -(m+s+\alpha k) \tau(s, m) v_{m+s+k} \\
& +(m+\beta s) \tau(s, m+k) v_{m+s+k} \\
& -(m+k+\alpha s) \tau(k, m) v_{m+k+s}
\end{aligned}
$$

Therefore the following condition on $\tau$ ensures that $M$ is a $W_{1}$-module.

$$
\begin{aligned}
(s-k) \tau(k+s, m)= & (m+\beta s) \tau(k, m+s)-(m+\beta k) \tau(s, m+k) \\
& +(m+s+\alpha k) \tau(s, m)-(m+k+\alpha s) \tau(k, m)
\end{aligned}
$$

Here we see that since these $\tau$-functions define the $W_{1}$-action, they define the module. Hence a classification of these $\tau$-functions will give an explicit classification of $W_{1}$-modules of this type. These $\tau$-functions also have a cohomological interpretation.

For a Lie algebra $L$, let $M$ be the $L$-module extension of the $L$-modules $\left(M_{1}, \rho_{1}\right),\left(M_{2}, \rho_{2}\right)$ given by

$$
0 \rightarrow M_{1} \rightarrow M \rightarrow M_{2} \rightarrow 0
$$

If we non-canonically identify $M_{2}$ with a subspace in $M$, the module action on $M$ can be given by

$$
\begin{aligned}
x . v & =\rho_{1}(x) v \\
x . w & =\rho_{2}(x) w+\tau(x, w)
\end{aligned}
$$

where $v \in M_{1}, w \in M_{2}$ and $\tau$ is a map $L \otimes M_{2} \rightarrow M_{1}$.

Lemma 3.1.2. The isomorphism classes of these module extensions $M$ are in one-to-one correspondence to the cohomology, $H^{1}\left(L, H_{0} m_{\mathbb{C}}\left(M_{2}, M_{1}\right)\right)$. 
Proof. By viewing the cohomology of $L$ with the module $\operatorname{Hom}\left(M_{2}, M_{1}\right)$ as in Chapter $2, C^{1}\left(L, \operatorname{Hom}\left(M_{2}, M_{1}\right)\right) \cong \operatorname{Hom}\left(L \otimes M_{2}, M_{1}\right)$ and 1-cocycles are elements in $\operatorname{ker} d_{1}$, where $d_{1}$ is defined as in (2.3.1) as

$$
d_{1}: C^{1}\left(L, \operatorname{Hom}\left(M_{2}, M_{1}\right)\right) \rightarrow C^{2}\left(L, \operatorname{Hom}\left(M_{2}, M_{1}\right)\right)
$$

Suppose that $\psi \in \operatorname{ker} d_{1}$. Then

$$
\begin{aligned}
0 & =\left(d_{1} \psi\right)(x, y) \\
& =\psi([x, y])-x \psi(y)+y \psi(x) \\
& =\psi([x, y]) w-(x \psi(y)) w+(y \psi(x)) w \\
& =\psi([x, y]) w-x(\psi(y) w)+\psi(y) x w+y(\psi(x) w)-\psi(x) y w \\
& =\psi([x, y], w)-x(\psi(y, w))+\psi(y, x w)+y(\psi(x, w))-\psi(x, y w)
\end{aligned}
$$

for all $x, y \in L$ and $w \in M_{2}$.

Then the module action on $M$ gives that

$$
\begin{aligned}
{[x, y] w } & =x(y w)-y(x w) \\
{[x, y] w+\tau([x, y], w) } & =x(y w+\tau(y, w)-y(x w+\tau(x, w)) \\
{[x, y] w+\tau([x, y], w) } & =x(y w)+\tau(x, y w)+x \tau(y, w)-y(x w)-\tau(y, x w)-y \tau(x, w)) \\
\Longrightarrow \tau([x, y], w) & =\tau(x, y w)+x \tau(y, w)-\tau(y, x w)-y \tau(x, w))
\end{aligned}
$$

Thus $\tau$ gives an extension of modules if and only if $\tau$ is a 1-cocycle.

By (2.3.1), 1-coboundaries are elements in the image of the map

$$
d_{0}: C^{0}\left(L, \operatorname{Hom}\left(M_{2}, M_{1}\right)\right) \rightarrow C^{1}\left(L, \operatorname{Hom}\left(M_{2}, M_{1}\right)\right)
$$


where $C^{0} \cong \operatorname{Hom}\left(M_{2}, M_{1}\right)$ and $C^{1} \cong \operatorname{Hom}\left(L \otimes M_{2}, M_{1}\right)$. Suppose that $\varphi \in \operatorname{Im}\left(d_{0}\right)$ so that $\varphi: L \otimes M_{2} \rightarrow M_{1}$. Then there exists $\bar{\varphi} \in \operatorname{Hom}\left(M_{2}, M_{1}\right)$, the preimage of $\varphi$ under $d_{0}$. The action of $d_{0}$ is given in 2.3.1) so that

$$
\left(d_{0} \bar{\varphi}\right)(x)=-x \varphi
$$

for all $x \in L$. Thus coboundaries in the space of $H^{1}\left(L, \operatorname{Hom}_{\mathbb{C}}\left(M_{2}, M_{1}\right)\right)$ are the functions $-x \varphi$ where $\varphi \in \operatorname{Hom}\left(L \otimes M_{2}, M_{1}\right)$.

Let us discuss when we have equivalent extensions. Equivalent extensions correspond to different liftings of $M_{2}$ into $M$ :

$$
w^{\prime}=w+\varphi(w)
$$

where $\varphi: M_{2} \rightarrow M_{1}$.

The $L$ action on $w^{\prime}$ becomes:

$$
\begin{aligned}
x . w^{\prime} & =\rho_{2}(x) w+\tau(x, w)+x \varphi(w) \\
& =\rho_{2}(x) w+\tau(x, w)+(x \varphi) x+\varphi(x w) \\
& =\rho_{2}(x) w^{\prime}+\tau(x, w)+(x \varphi)(w)
\end{aligned}
$$

As defined above, for $x \in W_{1}, v \in M_{1}, w$ in the preimage of $M_{2}$ in $M$ the $W_{1}$-action is

$$
\begin{aligned}
x . v & =\rho_{1}(x) v \\
x . w & =\rho_{2}(x) w+\tau(x, w)
\end{aligned}
$$

Thus, if there exists a change of basis such that the new 1-cocycle is zero, 
then $\tau(x, w)=-(x \varphi) w=-d_{0} \varphi(x, w)$, i.e. $\tau$ is a 1-coboundary. From here, we observe that two extensions are equivalent if the difference of their cocycles is a coboundary.

Thus, for $L=W_{1}, M_{1}=T(\alpha, \gamma), M_{2}=T(\beta, \gamma), \tau(k, m)$ is a 1-cocycle of the cohomology of $W_{1}$ with the module $\operatorname{Hom}(T(\beta, \gamma), T(\alpha, \gamma))$. As the extension is trivial if $\tau(k, m)=0$ then any cocycle equivalent to the zero cocycle will yield a trivial extension. This leads to a natural concept of a trivial cocycle.

Definition 3.1.3. A 1-cocycle is trivial if it is a 1-coboundary.

This condition turns out to be equivalent to saying that two $\tau$-functions are equivalent if for some change of basis, we can obtain one $\tau$-function from the other one. Since we are interested in modules and not cocycles themselves, a change of basis will not change our module and thus $\tau$-functions that can be obtained from a change of basis should be seen as equivalent.

\subsection{A new module extension}

Our final goal in this section is to obtain a basis for $M$ such that our cocycles will be polynomials in almost all cases. By this, we mean we want to find $\widehat{w}_{k}$ such that $\widehat{w}_{k} \rightarrow w_{k}$ under the map from $M \rightarrow T(\beta, \gamma)$ which will admit polynomial cocycles. The first thing to do is to lift our short exact sequence, or module extension, into the setting of $A W_{1}$-modules. To do this, we make use of the $A$-cover $\widehat{M}$ of $M$.

From Theorem 4.10 of [5], the $A$-cover of $M$ is cuspidal, so $\widehat{M}$ has finite dimensional weight spaces. We have the map $\pi: \widehat{M} \rightarrow M$ such that $\pi(\phi(x, u))=$ $\phi(x, u)(1)=x . u$. Define

$$
\widehat{M}(\alpha, \gamma)=\left\{\sum_{i} \phi\left(x_{i}, u_{i}\right) \mid \forall f \in A, \sum_{i}\left(f x_{i}\right) u_{i} \in T(\alpha, \gamma)\right\}
$$


Lemma 3.2.1. $\widehat{M} / \widehat{M}(\alpha, \gamma) \cong \widehat{T(\beta, \gamma)}$

Proof. Consider $\widehat{\pi}: \widehat{M} \rightarrow \widehat{T(\beta, \gamma)}$ by $\widehat{\pi}(\phi(x, u))=\phi(x, \bar{u})$, where $\bar{u}$ is the image of $u$ under the map that sends $M$ onto $T(\beta, \gamma)$. Clearly, this map is surjective as $\bar{M}=T(\beta, \gamma)$

Then for an arbitrary $\sum_{i} \phi\left(x_{i}, u_{i}\right) \in \widehat{M}$,

$$
\begin{aligned}
\sum_{i} \phi\left(x_{i}, u_{i}\right) \in \operatorname{ker} \widehat{\pi} & \left.\Longleftrightarrow \sum_{i} \phi\left(x_{i}, \bar{u}_{i}\right)=0, \text { in } \widehat{T(\beta, \gamma}\right) \\
& \Longleftrightarrow \forall f \in A, \sum_{i} \phi\left(x_{i}, \bar{u}_{i}\right)(f)=0 \\
& \Longleftrightarrow \forall f \in A, \sum_{i}\left(f x_{i}\right) \bar{u}_{i}=0 \\
& \Longleftrightarrow \forall f \in A, \overline{\sum_{i} f\left(x_{i}\right) u}=0 \\
& \Longleftrightarrow \forall f \in A, \sum_{i}\left(f x_{i}\right) u_{i} \in T(\alpha, \gamma) \\
& \Longleftrightarrow \sum_{i} \phi\left(x_{i}, u_{i}\right) \in \widehat{M}(\alpha, \gamma)
\end{aligned}
$$

So $\operatorname{ker} \widehat{\pi}=\widehat{M}(\alpha, \gamma)$

Lemma 3.2.2. $\widehat{\pi}$ is an homomorphism of $A W_{1}$-modules.

Proof. As $M \rightarrow T(\beta, \gamma)$ is a homomorphism of $W_{1}$-modules, then it is enough to show that $\widehat{\pi}$ is a homomorphism of $A$-modules.

Since $t^{s} \phi\left(e_{k}, u\right)\left(t^{m}\right)=e_{k+s+m} u=\phi\left(t^{s} e_{k}, u\right)\left(t^{m}\right)$, it follows from (2.2.5) that $(f \phi)(x, u)=\phi(f x, u)$ for all $f \in A, x \in W_{1}, u \in M$. Then:

$$
\begin{aligned}
f \widehat{\pi}(\phi(x, u)) & =f \phi(x, \bar{u}) \\
& =\phi(f x, \bar{u}) \\
& =\widehat{\pi}(\phi(f x, u)) \\
& =\widehat{\pi}(f \phi(x, u)
\end{aligned}
$$


so that the $A$-action is preserved by $\widehat{\pi}$.

It follows from this Lemma that $\widehat{M}(\alpha, \gamma)$ is an $A W_{1}$-module as it is the kernel of a homomorphism of $A W_{1}$-modules. We obtain the short exact sequence

$$
0 \rightarrow \widehat{M}(\alpha, \gamma) \rightarrow \widehat{M} \rightarrow \widehat{T(\beta, \gamma)} \rightarrow 0
$$

We want to be able to relate this module back to $T(\beta, \gamma)$ instead of $\widehat{T(\beta, \gamma)}$ and this new module extension will turn out to be too large. Instead, we will find a submodule $\widetilde{M}$ of $\widehat{M}$ that will admit a short exact sequence with $T(\beta, \gamma) \subset \widehat{T(\beta, \gamma)}$.

The next two lemmas will help us relate $\widehat{T(\beta, \gamma)}$ to $T(\beta, \gamma)$. Define $\varepsilon_{j}$ and $\eta_{j}$ in $\operatorname{Hom}(A, T(\beta, \gamma))$ by

$$
\begin{aligned}
\varepsilon_{j}\left(t^{m}\right) & =w_{m+j} \\
\eta_{j}\left(t^{m}\right) & =(m+j) w_{m+j}
\end{aligned}
$$

Lemma 3.2.3. $\varepsilon_{j} \in \widehat{T(\beta, \gamma)}$ for all $j \in \gamma+\mathbb{Z}, \beta \neq 1$ and $\eta_{j} \in \widehat{T(\beta, \gamma)}$ for all $j \in \gamma+\mathbb{Z}, \beta \neq 0$.

Proof. For $\beta \neq 1$, consider

$$
\left.\frac{1}{1-\beta}\left(\phi\left(e_{0}, w_{j}\right)-\phi\left(e_{1}, w_{j-1}\right)\right) \in \widehat{T(\beta, \gamma}\right)
$$

where $w_{j}, w_{j-1} \in T(\beta, \gamma), j \in \gamma+\mathbb{Z}$.

Note that:

$$
\begin{aligned}
\frac{1}{1-\beta}\left(\phi\left(e_{0}, w_{j}\right)-\phi\left(e_{1}, w_{j-1}\right)\right)\left(t^{m}\right) & =\frac{1}{1-\beta}\left(\left(t^{m} e_{0}\right) \cdot w_{j}-\left(t^{m} e_{1}\right) \cdot w_{j-1}\right) \\
& =\frac{1}{1-\beta}\left(e_{m} \cdot w_{j}-e_{m+1} \cdot w_{j-1}\right)
\end{aligned}
$$




$$
\begin{aligned}
& =\frac{1}{1-\beta}(j+\beta m-j+1-\beta(m+1)) w_{j+m} \\
& =\frac{1}{1-\beta}(1-\beta) w_{j+m} \\
& =w_{j+m}
\end{aligned}
$$

So that, as long as $\beta \neq 1, \varepsilon_{j} \in \widehat{T(\beta, \gamma)}$ for all $j \in \gamma+\mathbb{Z}$.

For $\eta_{j}$, first notice that the action of $\phi\left(e_{k}, w_{j}\right)$ on $t^{m}$ is given by:

$$
\psi\left(e_{k}, w_{j}\right)\left(t^{m}\right)=\beta \eta_{j+k}\left(t^{m}\right)+(1-\beta) j \varepsilon_{j+k}\left(t^{m}\right)
$$

Thus for $\beta \neq 0, \eta_{j}=\frac{1}{\beta}\left(\phi\left(e_{k}, w_{j-k}\right)-(1-\beta)(j-k) \varepsilon_{j}\right)$ and so $\left.\eta_{j} \in \widehat{T(\beta, \gamma}\right)$ as long as $\beta \neq 0$.

Remark 3.2.4. The equation 3.2 .1 tells us that $\widehat{T(\beta, \gamma)}$ is spanned by the vectors $\varepsilon_{j}, \eta_{j}$ for $j \in \gamma+\mathbb{Z}$ when $\beta \neq 1$, and $\beta \neq 0$. In the special cases of $\beta=0$ or $\beta=1$, $\widehat{T(\beta, \gamma)}$ is spanned by vectors $\varepsilon_{j}$ or $\eta_{j}$ respectively.

Lemma 3.2.5. Let $\beta \neq 1$. The subspace of $\widehat{T(\beta, \gamma)}$ spanned by $\left\{\varepsilon_{i} \mid i \in \gamma+\mathbb{Z}\right\}$ is isomorphic to $T(\beta, \gamma)$.

Proof. The action of $W_{1}$ on $\varepsilon_{m}$ is given by:

$$
e_{k} \cdot \varepsilon_{m}=(m+\beta k) \varepsilon_{m+k}
$$

so that $\left\{\varepsilon_{i} \mid i \in \gamma+\mathbb{Z}\right\}$ is a submodule of $\widehat{T(\beta, \gamma)}$.

Then, as long as $\beta \neq 1$, the map $\varepsilon_{i} \rightarrow w_{i}$ is surjective and thus the claim follows.

Consequently, we will denote $\operatorname{span}\left\{\varepsilon_{j} \mid j \in \mathbb{Z}\right\}$ by $T(\beta, \gamma)$, and view $T(\beta, \gamma)$ as a subspace of $\widehat{T(\beta, \gamma)}$ when $\beta \neq 1$. 
Now we define $\widetilde{M}=\widehat{\pi}^{-1}(T(\beta, \gamma))$. As $T(\beta, \gamma)$ contains the zero element of $\widehat{T(\beta, \gamma)}, \widehat{M}(\alpha, \gamma) \subseteq \widetilde{M}$. Also $T(\beta, \gamma)$ is an $A W_{1}$-submodule of $\widehat{T(\beta, \gamma)}$ so that, as $\widetilde{M}$ is the homomorphic preimage of an $A W_{1}$-module, it is itself an $A W_{1}$-module. From here, we have the first half of our short exact sequence. The only thing we need is to find the module $\widetilde{M} / \widehat{M}(\alpha, \gamma)$.

Lemma 3.2.6. $\pi: \widetilde{M} \rightarrow M$ is surjective when $\beta \neq 1$ and $(\alpha \neq 1$ or $\gamma \notin \mathbb{Z})$.

Proof. Notice that $\pi: \widehat{T(\alpha, \gamma)} \rightarrow T(\alpha, \gamma)$ will be surjective if $\pi(\widehat{T(\alpha, \gamma)})=$ $W_{1} T(\alpha, \gamma)=T(\alpha, \gamma)$ (by proposition 4.5 in [5]). This happens if and only if $\alpha \neq 1$ or $\gamma \notin \mathbb{Z}$ :

$$
\text { For } k \neq 0, e_{0} \cdot v_{k}=k v_{k} \Longrightarrow \frac{1}{k} e_{0} \cdot v_{k}=v_{k}
$$

For $k=0, e_{s} \cdot v_{-s}=(-s+\alpha s) v_{0} \Longrightarrow v_{0} \in \operatorname{Im} \pi \Longleftrightarrow \alpha \neq 1$ or $\gamma \notin \mathbb{Z}$

Then $\pi: \widehat{T(\alpha, \gamma)} \rightarrow T(\alpha, \gamma)$ is surjective unless $\alpha=1$ and $\gamma \in \mathbb{Z}$ so by extending this map, $\widehat{M}(\alpha, \gamma) \rightarrow T(\alpha, \gamma)$ is also surjective. It is left to show that $\widehat{\pi}: \widetilde{M} / \widehat{M}(\alpha, \gamma) \rightarrow T(\beta, \gamma)$ is surjective.

By Lemma 3.2.1, $\widehat{\pi}: \widehat{M} \rightarrow \widehat{T(\beta, \gamma)}$ is surjective. As $T(\beta, \gamma) \subseteq \widehat{T(\beta, \gamma)}$ for $\beta \neq 1$ and $\widetilde{M}=\widehat{\pi}^{-1}(T(\beta, \gamma))$, then it follows that $\widehat{\pi}: \widetilde{M} \rightarrow T(\beta, \gamma)$ is surjective.

When $\alpha=1, \pi$ will be surjective onto the extension given by the submodule of $T(1,0)$ :

$$
0 \rightarrow T^{\circ}(1,0) \rightarrow M^{\prime} \rightarrow T(\beta, 0)
$$

As we will see at the end of the chapter, these extensions will can be easily extended to the original extension $M$. 
Now, for $\beta \neq 1$, we have the short exact sequence

$$
0 \rightarrow \widehat{M}(\alpha, \gamma) \rightarrow \widetilde{M} \rightarrow T(\beta, \gamma) \rightarrow 0
$$

Here, all these modules have are cuspidal; this follows as $\widehat{M}(\alpha, \gamma)$ and $\widetilde{M}$ are $A W_{1}$-submodules of $\widehat{M}$. By definition, $T(\beta, \gamma)$ has 1-dimensional weight spaces.

\subsection{Submodules of $\operatorname{Hom}(A, T(\alpha, \gamma))$ with finite dimensional weight spaces}

The next proposition will be very important in showing that for a general case, 1-cocycles are polynomial functions. First, introduce the maps in $\operatorname{Hom}(A, T(\alpha, \gamma))$ for $k \in \gamma+\mathbb{Z}, i \in \mathbb{Z}_{+}$,

$$
\theta_{k}^{(i)}: t^{m} \rightarrow \frac{(m+k)^{i}}{i !} v_{m+k}
$$

and

$$
\delta_{k}: t^{m} \rightarrow \begin{cases}v_{m+k}, & m=-k \\ 0, & m \neq-k\end{cases}
$$

Remark 3.3.1. When $\alpha=\beta, \theta_{k}^{(0)}=\varepsilon_{k}$ and $\theta_{k}^{(1)}=\eta_{k}$. We make the distinction between these functions for convenience of notation later on.

Proposition 3.3.2. Any $A W_{1}$-submodule in $\operatorname{Hom}(A, T(\alpha, \gamma))$ with finite dimensional weight spaces is contained in a submodule spanned by:

1. $\left\{\theta_{k}^{(0)}, \cdots, \theta_{k}^{(N)} \mid k \in \gamma+\mathbb{Z}\right\}$ for some $N \in \mathbb{N}$, when $\alpha \neq 0$

2. $\left\{\theta_{k}^{(0)}, \cdots, \theta_{k}^{(N)}, \delta_{k} \mid k \in \gamma+\mathbb{Z}\right\}$ for some $N \in \mathbb{N}$, when $\alpha=0$

Proof. Suppose that $\varphi \in \operatorname{Hom}(A, T(\alpha, \gamma))$. Without loss of generality, suppose 
that $\varphi$ is an element of weight $k$ and $\varphi\left(t^{m}\right)=a_{m} v_{m+k}$. We will show that the function $a(m)=a_{m}$ is a polynomial in $m$ when $\alpha \neq 0$.

First, we consider $t^{-i} e_{i} \varphi \in \operatorname{Hom}(A, T(\alpha, \gamma))$. In this notation, $t^{-i} e_{i} \varphi=$ $t^{-i}\left(e_{i} \varphi\right)$ which is not the same as $\left(t^{-i} e_{i}\right) \varphi$. Since $e_{i} \varphi$ is an element with weight $k+i$, and $t^{-i}$ is an element of weight $-i, t^{-i} e_{i} \varphi$ is still an element of weight $k$ in $\operatorname{Hom}(A, T(\alpha, \gamma))$. Then $\left(t^{-i} e_{i} \varphi\right)\left(t^{m}\right)=b_{m} v_{m+k}=b(m) v_{m+k}$. The functions $a$ and $b$ are connected by the following relation.

$$
\begin{aligned}
b_{m} v_{m+k} & =\left(t^{-i} e_{i} \varphi\right)\left(t^{m}\right) \\
& =\left(e_{i} \varphi\right)\left(t^{m-i}\right) \\
& =e_{i}\left(\varphi\left(t^{m-i}\right)\right)-\varphi\left(e_{i} t^{m-i}\right) \\
& =e_{i}\left(a_{m-i} v_{m-i+k}\right)-\varphi\left((m-i) t^{m-i-1+i+1}\right) \\
& =a_{m-i}\left(e_{i} v_{m-i+k}\right)-(m-i) \varphi\left(t^{m}\right) \\
& =(m-i+k-\alpha i) a_{m-i} v_{m+k}-(m-i) a_{m} v_{m+k} \\
\Longrightarrow b_{m} & =(m-i+k-\alpha i) a_{m-i}-(m-i) a_{m}
\end{aligned}
$$

So that

$$
b(m)=m(a(m-i)-a(m))+(k-i-\alpha i) a(m-i)+i a(m)
$$

Define an action of $t^{-i} e_{i}$ on $a$ as

$$
\left(t^{-i} e_{i} a\right)(m)=m(a(m-i)-a(m))+(k-i-\alpha i) a(m-i)+i a(m)
$$

Now define:

$$
\begin{gathered}
z_{n}=\sum_{i=0}^{n+1}(-1)^{i}\left(\begin{array}{c}
n+1 \\
i
\end{array}\right) t^{i-1} e_{1-i} \\
y_{n}=\sum_{i=0}^{n}(-1)^{i}\left(\begin{array}{c}
n \\
i
\end{array}\right) t^{i} e_{-i}
\end{gathered}
$$


Lemma 3.3.3. $z_{n}=z_{n-1}-y_{n}$ for all $n \in \mathbb{N}$.

Proof.

$$
\begin{aligned}
z_{n-1}-y_{n} & =\sum_{i=0}^{n}(-1)^{i}\left(\begin{array}{c}
n \\
i
\end{array}\right) t^{i-1} e_{1-i}-\sum_{i=0}^{n}(-1)^{i}\left(\begin{array}{c}
n \\
i
\end{array}\right) t^{i} e_{-i} \\
& =t^{-1} e_{1}+\sum_{i=0}^{n-1}(-1)^{i+1}\left(\begin{array}{c}
n \\
i+1
\end{array}\right) t^{i} e_{-i}+\sum_{i=0}^{n-1}(-1)^{i+1}\left(\begin{array}{c}
n \\
i
\end{array}\right) t^{i} e_{-i}+(-1)^{n+1} t^{n} e_{-n} \\
& =t^{-1} e_{1}+\sum_{i=0}^{n-1}(-1)^{i+1}\left(\begin{array}{c}
n+1 \\
i+1
\end{array}\right) t^{i} e_{-i}+(-1)^{n+1} t^{n} e_{-n} \\
& =\sum_{i=0}^{n+1}(-1)^{i}\left(\begin{array}{c}
n+1 \\
i
\end{array}\right) t^{i-1} e_{1-i} \\
& =z_{n}
\end{aligned}
$$

We can give an explicit form of $z_{n}$ and $y_{n}$ by the following

$$
\begin{gathered}
\left(z_{n} a\right)(m)=\sum_{i=0}^{n+1}(-1)^{i}\left(\begin{array}{c}
n+1 \\
i
\end{array}\right)(m(a(m+i-1)-a(m)) \\
\quad+(k-1+i-\alpha+\alpha i) a(m+i-1)+(1-i) a(m)) \\
\left(y_{n} a\right)(m)=\sum_{i=0}^{n}(-1)^{i}\left(\begin{array}{c}
n \\
i
\end{array}\right)(m(a(m+i)-a(m)) \\
+(k+i+\alpha i) a(m+i)-i a(m))
\end{gathered}
$$

Since $\sum_{i=0}^{j}(-1)^{i}\left(\begin{array}{l}j \\ i\end{array}\right)=0$ and $\sum_{i=0}^{j}(-1)^{i}\left(\begin{array}{l}j \\ i\end{array}\right) i=0$, this simplifies to

$$
\begin{aligned}
& \left(z_{n} a\right)(m)=\sum_{i=0}^{n+1}(-1)^{i}\left(\begin{array}{c}
n+1 \\
i
\end{array}\right)(m+k+(\alpha+1)(i-1)) a(m+i-1) \\
& \left(y_{n} a\right)(m)=\sum_{i=0}^{n}(-1)^{i}\left(\begin{array}{c}
n \\
i
\end{array}\right)(m+k+i+\alpha i) a(m+i)
\end{aligned}
$$


Observe that $a$ is a function from $\gamma+\mathbb{Z}$ to $\mathbb{C}$. By assumption, $\varphi$ in contained in an $A W_{1}$-module with finite dimensional weight spaces.

By Lemma 4 of [3], $\left[z_{-1}, z_{n}\right]=-n z_{n}$ for all $n \in \mathbb{N}$, which means that $z_{n}$ will be an eigenvector of $a d z_{-1}$. As $z_{n} \in M_{\ell \times \ell}$ for some $\ell \in \mathbb{N}, a d z_{-1} \in M_{\ell^{2} \times \ell^{2}}(\mathbb{C})$. Thus $a d z_{-1}$ has at most $\ell^{2}$ unique eigenvalues, so that $z_{n}$ can only be non-zero for finitely many $n \in \mathbb{N}$.

Let $\bar{n}=\max \left\{n \in \mathbb{N} \mid z_{n} \neq 0\right\}$ if this set is non-empty, and set $\bar{n}=1$ if the set is empty. Then $z_{n}=0$ for all $n>\bar{n}$, then $z_{n+1}$ is also zero for all $n>\bar{n}$, so $z_{n}-z_{n+1}$ is zero for all $n>\bar{n}$. Thus $y_{n+1}$ is also zero. By shifting $m$ to $m-1$, we observe

$$
\left(y_{n+1} a\right)(m)=\sum_{i=0}^{n+1}(-1)^{i}\left(\begin{array}{c}
n+1 \\
i
\end{array}\right)(m+k+(\alpha+1) i-1) a(m+i-1)
$$

and $y_{n+1}$ is still the zero function. So

$$
\begin{aligned}
(0 \cdot a)(m)= & \left(\left(z_{n}-y_{n+1}\right) \cdot a\right)(m) \\
= & \sum_{i=0}^{n+1}(-1)^{i}\left(\begin{array}{c}
n+1 \\
i
\end{array}\right)(m a(m+i-1)+(k-1+i-\alpha+\alpha i) a(m+i-1) \\
& -m a(m+i-1)-(k+i+\alpha i-1) a(m+i-1)) \\
= & \sum_{i=0}^{n+1}(-1)^{i}\left(\begin{array}{c}
n+1 \\
i
\end{array}\right)(k-1+i-\alpha+\alpha i-k+i+\alpha i-1) a(m+i-1) \\
= & -\sum_{i=0}^{n+1}(-1)^{i}\left(\begin{array}{c}
n+1 \\
i
\end{array}\right) \alpha a(m+i-1)
\end{aligned}
$$

So, as long as $\alpha \neq 0$,

$$
0=\sum_{i=0}^{n+1}(-1)^{i}\left(\begin{array}{c}
n+1 \\
i
\end{array}\right) a(m+i-1)
$$

for all $n>\bar{n}$ and hence $\left\{a_{m}\right\}$ satisfy recurrence relations. 
This in turn tells us that each $a$ is a polynomial in $m$ by the use of Lemma 3 in [3], so that $a(m)$ is in the $\operatorname{span}(m+k)^{i}$ for $i=1, \ldots, N$. Then it follows that $\varphi$ is in the span of $\left\{\theta_{m}^{(0)}, \cdots, \theta_{m}^{(N)}\right\}$ for some $N \in \mathbb{N}$, where $N=\operatorname{deg}(a)$.

If $\alpha=0$, then

$$
\left(z_{n} a\right)(m)=\sum_{i=0}^{n+1}(-1)^{i}\left(\begin{array}{c}
n+1 \\
i
\end{array}\right)(m+i-1+k) a(m+i-1)
$$

Set $d(m)=(m+k) a(m)$ so that

$$
\left(z_{n} a\right)(m)=\sum_{i=0}^{n+1}(-1)^{i}\left(\begin{array}{c}
n+1 \\
i
\end{array}\right) d(m+i-1)
$$

By the previous argument, $d$ is a polynomial in $m$ with a root at $-k$. So there exists a polynomial $g(m)$ such that $d(m)=(m+k) g(m)$. Thus, $a(m)$ and $g(m)$ agree on every integer except $-k$ so that

$$
a(m)=g(m)+c \delta_{m,-k}
$$

for some $c \in \mathbb{C}$, where $g(m)=\frac{d(m)}{m+k}$ is a polynomial in $m$.

This suggests that it is possible that $a$ could have a delta-function component. It needs to be shown that if $\varphi\left(t^{m}\right)=\delta_{m,-k} v_{m+k}$, then $\varphi$ is contained in some $A W_{1}$-module with finite dimensional weight spaces, and so this case exists.

Define $f_{k}\left(t^{m}\right)=\delta_{m,-k} v_{m+k}$. Then

$$
f_{k}\left(t^{m}\right)= \begin{cases}v_{0} & , m=-k \\ 0 & , m \neq-k\end{cases}
$$

It is enough to show that $\left\{f_{k} \mid k \in \gamma+\mathbb{Z}\right\}$ as an $A W_{1}$-module has finite dimensional weight spaces. 
The $A$ action on $f_{k}$ :

$$
\begin{aligned}
\left(t^{\ell} f_{k}\right)\left(t^{m}\right) & =f_{k}\left(t^{m+\ell}\right) \\
& =\delta_{m+\ell,-k} v_{0} \\
& =\delta_{m,-k-\ell} v_{0} \\
& =f_{k+\ell}\left(t^{m}\right)
\end{aligned}
$$

So $t^{\ell} f_{k}=f_{k+\ell}$ is the $A$ action for all $\ell \in \mathbb{Z}$.

The $W_{1}$ action on $f_{s}$ :

$$
\begin{aligned}
\left(e_{\ell} f_{k}\right)\left(t^{m}\right) & =e_{\ell}\left(f_{k}\left(t^{m}\right)\right)-f_{k}\left(e_{\ell} t^{m}\right) \\
& =\delta_{m,-k} e_{\ell} v_{0}-f_{k}\left(m t^{m+l \ell}\right) \\
& =-m \delta_{m+\ell,-k} v_{0} \\
& =(k+\ell) \delta_{m,-k-\ell} v_{0} \\
& =f_{k+\ell} v_{0}
\end{aligned}
$$

So $e_{\ell} f_{k}=(k+\ell) f_{k+\ell}$ is the $W_{1}$ action for all $\ell \in \mathbb{Z}$.

This shows us that this space has 1-dimensional weight spaces and hence, finite dimensional weight spaces.

\subsection{Finding an appropriate basis for $M$}

From here, we would like to obtain a basis of $M$ that will admit polynomial cocycles. In particular, we would like to make use of the last proposition to show that for $\beta \neq 1$, we can obtain a basis of $M$ so that all possible 1-cocycles are of 
the form described in the past proposition.

Let $\mathscr{L}$ be the Lie algebra spanned by the elements $z^{i} \frac{d}{d z}$ where $i \in \mathbb{Z}$. Denote $\mathscr{L}_{+}$to be the subalgebra spanned by the elements $z^{i} \frac{d}{d z}$ where $i \in \mathbb{Z}$ and $i \geq 1$.

Theorem 3.4.1 (Theorem 4.11 of [5]]). If $V$ is a cuspidal $A W_{1}$-module with weights in $\gamma+\mathbb{Z}$ for some $\gamma \in \mathbb{C}$, then there exists a finite dimensional module $(U, \rho)$ of $\mathscr{L}_{+}$such that

$$
V \cong A \otimes U
$$

and with $W_{1}$-action given by

$$
e_{k}\left(t^{m} \otimes u\right)=(m+\gamma) t^{m+k} \otimes u+\sum_{i=1}^{\infty} \frac{k^{i}}{i !} t^{m+k} \otimes \rho\left(z^{i} \frac{d}{d z}\right) u
$$

for all $k \in \mathbb{Z}, m \in \mathbb{Z}, u \in U$.

We will assume for the rest of the chapter that $\beta \neq 1$. We will make use of this theorem first on $\widehat{T(\beta, \gamma)}$ and later on $\widetilde{M}$. As shown in the last section, $\widehat{T(\beta, \gamma)}$ is spanned by the basis vectors $\varepsilon_{j}, \eta_{j}$ for all $j \in \gamma+\mathbb{Z}$ when $\beta \neq 0$ and spanned just by $\varepsilon_{j}$ for $\beta=0$. By computing the action using 2.2 .2 , the $W_{1}$-action on these elements is given by:

$$
\begin{aligned}
& e_{k} \varepsilon_{j}=(j+\beta k) \varepsilon_{j+k} \\
& e_{k} \eta_{j}=(j+k(\beta-1)) \eta_{j+k}-k^{2}(\beta-1) \varepsilon_{j+k}
\end{aligned}
$$

If $\varepsilon_{j}=t^{j} \otimes \varepsilon, \eta_{j}=t^{j} \otimes \eta$, then $\widehat{T(\beta, \gamma)} \cong A \otimes U^{\prime}$, where $U^{\prime}=\langle\varepsilon, \eta\rangle$. By the previous theorem, the $W_{1}$ action of $A \otimes U^{\prime}$ is given by

$$
e_{k}\left(t^{j} \otimes u\right)=t^{j+k} \otimes\left(j u+\sum_{i=1}^{\infty} \frac{k^{i}}{i !} \rho\left(z^{i} \frac{d}{d z}\right) u\right)
$$


so it is possible to derive the representation $\rho$ :

$$
\begin{array}{ll}
\rho\left(z \frac{d}{d z}\right) \varepsilon=\beta \varepsilon, & \rho\left(z^{i} \frac{d}{d z}\right) \varepsilon=0, \forall i \geq 2 \\
\rho\left(z \frac{d}{d z}\right) \eta=(\beta-1) \eta, & \rho\left(z^{2} \frac{d}{d z}\right) \eta=-2(\beta-1) \varepsilon \\
\rho\left(z^{i} \frac{d}{d z}\right) \eta=0, \forall i \geq 3 &
\end{array}
$$

We can then write $\rho$ as a matrix as we know its action on the basis of $\widehat{T(\beta, \gamma)}$.

$$
\begin{aligned}
\rho\left(z \frac{d}{d z}\right) & =\left(\begin{array}{cc}
\beta & 0 \\
0 & \beta-1
\end{array}\right), \quad \rho\left(z^{2} \frac{d}{d z}\right)=\left(\begin{array}{cc}
0 & -2(\beta-1) \\
0 & 0
\end{array}\right) \\
\rho\left(z^{i} \frac{d}{d z}\right) & =0, \forall i \geq 3 .
\end{aligned}
$$

Define $\sigma_{0} \in \widehat{M}$ such that

$$
\sigma_{0}=\frac{1}{1-\beta}\left(\phi\left(e_{0}, w_{\gamma}\right)-\phi\left(e_{1}, w_{\gamma-1}\right)\right)
$$

where $w_{\gamma-1}, w_{\gamma} \in M$, the preimages of the basis vectors $w_{\gamma-1}, w_{\gamma} \in T(\beta, \gamma)$ as at the start of this chapter. Then

$$
\begin{aligned}
\widehat{\pi}\left(\sigma_{0}\right) & =\frac{1}{1-\beta}\left(\phi\left(e_{0}, \bar{w}_{\gamma}\right)-\phi\left(e_{1}, \bar{w}_{\gamma-1}\right)\right) \\
& =\varepsilon_{\gamma}
\end{aligned}
$$

Thus $\sigma_{0} \in \widehat{\pi}^{-1}(T(\beta, \gamma))=\widetilde{M}$.

Let $\sigma_{m}=t^{m} \sigma_{0}$ and define $\widehat{w}_{m}=\pi\left(\sigma_{m}\right) \in M$. It follows that

$$
\widehat{w}_{m}=\pi\left(\sigma_{m}\right)
$$




$$
\begin{aligned}
& =\sigma_{m}(1) \\
& =\left(t^{m} \sigma_{0}\right)(1) \\
& =\sigma_{0}\left(t^{m}\right)
\end{aligned}
$$

As we have already concluded, $\widetilde{M}$ is a submodule of a cuspidal $A W_{1}$-module, and thus itself a cuspidal $A W_{1}$-module. Thus, we can write $\widetilde{M}=A \otimes \widetilde{M}_{\gamma}$, and by Theorem 3.4 .1 the $\gamma$-weight space $\widetilde{M}_{\gamma}$ admits the action of $\mathscr{L}_{+}$. As shown below, $\sigma_{0} \in \widetilde{M}_{\gamma}$ so that $\sigma_{0}=1 \otimes \sigma$.

$$
\begin{aligned}
e_{0} \sigma_{0}\left(t^{m}\right) & =e_{0}\left(\sigma\left(t^{m}\right)\right)-\sigma_{0}\left(e_{0} t^{m}\right) \\
& \left.=\frac{1}{\beta-1} e_{0} \cdot\left(e_{m} \cdot w_{\gamma}-e_{m+1} w_{\gamma-1}\right)\right)-m \sigma_{0}\left(t^{m}\right) \\
& =\gamma \sigma_{0}\left(t^{m}\right)
\end{aligned}
$$

Then

$$
\begin{aligned}
e_{k} \sigma_{0} & =e_{k}(1 \otimes \sigma)=t^{k} \otimes\left(\sum_{i=1}^{\infty} \frac{k^{i}}{i !} \rho\left(z^{i} \frac{d}{d z}\right) \sigma\right) \\
& =t^{k} \otimes\left(k u_{1}+k^{2} u_{2}+\cdots+k^{n} u_{n}\right)
\end{aligned}
$$

for all $k \in \mathbb{Z}$ where $u_{i}=\frac{1}{i !} \rho\left(z^{i} \frac{d}{d z}\right) \sigma$. As $\sigma \in \widetilde{M}_{\gamma}$, each $u_{i} \in \widetilde{M}_{\gamma}$. Then $e_{k} \sigma_{0} \in \widetilde{M}_{k+\gamma}$.

Then

$$
\begin{aligned}
e_{k} \widehat{w}_{m} & =e_{k}\left(\sigma_{0}\left(t^{m}\right)\right) \\
& =\left(e_{k} \sigma_{0}\right)\left(t^{m}\right)+\sigma_{0}\left(e_{k} t^{m}\right) \\
& =\left(t^{k} \otimes\left(k u_{1}+k^{2} u_{2}+\cdots+k^{n} u_{n}\right)\right)\left(t^{m}\right)+\sigma_{0}\left(m t^{k+m}\right) \\
& =\left(1 \otimes\left(k u_{1}+k^{2} u_{2}+\cdots+k^{n} u_{n}\right)\right)\left(t^{m+k}\right)+m \sigma_{0}\left(t^{k+m}\right) \\
& =(m+\beta k) \widehat{w}_{k+m}+\zeta(k, m) \widehat{w}_{k+m}+\tau(k, m) v_{k+m}
\end{aligned}
$$


We would like to show that $\zeta$ is the zero function and

$$
e_{k} \widehat{w}_{m}=(m+\beta k) \widehat{w}_{k+m}+\tau(k, m) v_{k+m}
$$

where $\tau(k, m)$ is a polynomial function in $k$ and $m$.

Recall that $\widehat{\pi}: \widetilde{M} \rightarrow T(\beta, \gamma)$ is a homomorphism with $\operatorname{ker}(\widehat{\pi})=\widehat{M}(\alpha, \gamma)$, and $\widetilde{M} / \widehat{M}(\alpha, \gamma) \cong T(\beta, \gamma)$. As was proved before, $\widehat{\pi}$ is an $A W_{1}$-module homomorphism from $\widetilde{M} \cong A \otimes \widetilde{M}_{\gamma} \rightarrow A \otimes \widehat{T(\beta, \gamma)} \cong \widehat{T(\beta, \gamma)}$.

From here our main motivation is to make use of both Proposition 3.3 .2 and equation (3.4.2). The problem is that the first result is in the context of $\operatorname{Hom}(A, T(\alpha, \gamma))$ where as the second result is in the context of $\mathscr{L}_{+}$-modules. The next two lemmas will give us a way to relate $A W_{1}$-modules to $\mathscr{L}_{+}$-modules.

Lemma 3.4.2. Suppose $\varphi: A \otimes X \rightarrow A \otimes Y$ is a homomorphism of $A W_{1}$-modules for some $\mathscr{L}+$-modules $X, Y$. Then the restriction map $\varphi^{\prime}: 1 \otimes X \rightarrow 1 \otimes Y$ is a homomorphism of $\mathscr{L}_{+}$-modules.

Proof. First notice that since $\varphi$ is an $A$-module homomorphism and a $W_{1}$-module homomorphism,

$$
\begin{gathered}
\varphi\left(t^{m} \otimes x\right)=t^{m} \cdot \varphi(1 \otimes x)=t^{m} \otimes \varphi^{\prime}(x) \\
\varphi\left(e_{k}\left(t^{m} \otimes x\right)\right)=e_{k}\left(\varphi\left(t^{m} \otimes x\right)\right)
\end{gathered}
$$

From Theorem 3.4.1.

$$
e_{k}\left(t^{m} \otimes x\right)=t^{m+k} \otimes\left(m x+\sum_{i=0}^{\infty} \frac{k^{i}}{i !} \rho\left(z^{i} \frac{d}{d z}\right) x\right)
$$


so that the left-hand side of 3.4 .3 becomes

$\varphi\left(t^{m+k} \otimes\left(m x+\sum_{i=0}^{\infty} \frac{k^{i}}{i !} \rho\left(z^{i} \frac{d}{d z}\right) x\right)\right)=t^{m+k} \otimes \varphi^{\prime}\left(m x+\sum_{i=0}^{\infty} \frac{k^{i}}{i !} \rho\left(z^{i} \frac{d}{d z}\right) x\right)$

The right-hand side of 3.4 .3 becomes

$$
e_{k}\left(t^{m} \otimes \varphi^{\prime}(x)\right)=t^{m+k} \otimes\left(m \varphi^{\prime}(x)+\sum_{i=0}^{\infty} \frac{k^{i}}{i !} \rho\left(z^{i} \frac{d}{d z}\right) \varphi^{\prime}(x)\right)
$$

Then the following is true for all $k \in \mathbb{Z}$ :

$$
\varphi^{\prime}\left(\left(\sum_{i=0}^{\infty} \frac{k^{i}}{i !} \rho\left(z^{i} \frac{d}{d z}\right)\right) x\right)=\left(\left(\sum_{i=0}^{\infty} \frac{k^{i}}{i !} \rho\left(z^{i} \frac{d}{d z}\right)\right) \varphi^{\prime}(x)\right)
$$

These will be polynomial functions since for all $x \in \mathscr{L}_{+}$, there exists $n \in \mathbb{N}$ such that $\rho\left(z^{i} \frac{d}{d z}\right) x=0$. Since these functions are equal on all integer values then necessarily they must be equal as polynomial functions. Therefore,

$$
\varphi^{\prime}\left(\rho\left(z^{i} \frac{d}{d z}\right) x\right)=\rho\left(z^{i} \frac{d}{d z}\right) \varphi^{\prime}(x), \forall i \in \mathbb{Z}, \forall x \in X
$$

Thus, $\varphi^{\prime}$ is a homomorphism of $\mathscr{L}_{+}$-modules.

Now, by defining $\left.\widehat{\pi}^{\prime}: 1 \otimes \widetilde{M}_{\gamma} \rightarrow 1 \otimes \widehat{T(\beta, \gamma)}\right)_{\gamma}$, by the above results, $\widehat{\pi}^{\prime}$ is a homomorphism of $\mathscr{L}_{+}$-modules so that $\widehat{\pi}^{\prime} \rho=\rho^{\prime}$ for the action $\rho$ of $\mathscr{L}_{+}$on $U$. $\widehat{\pi}^{\prime}$ is surjective and we will now determine the kernel.

Lemma 3.4.3. $\widehat{M}(\alpha, \gamma)=A \otimes \operatorname{ker}\left(\widehat{\pi}^{\prime}\right)$

Proof. Since $\widehat{M}(\alpha, \gamma)$ is the kernel of a homomorphism of $A W_{1}$-modules, then it is itself an $A W_{1}$-module. Therefore, $\widehat{M}(\alpha, \gamma) \cong A \otimes V$ for some $\mathscr{L}_{+}$-module $V$. 
Take $t^{m} \otimes v \in \widehat{M}(\alpha, \gamma)$. Then $\widehat{\pi}\left(t^{m} \otimes v\right)=0$ since $\widehat{M}(\alpha, \gamma)=\operatorname{ker}(\widehat{\pi})$. So

$$
\begin{aligned}
0 & =\widehat{\pi}\left(t^{m} \otimes v\right) \\
& =t^{m} \otimes \widehat{\pi}^{\prime}(v) \\
\Longrightarrow \widehat{\pi}^{\prime}(v) & =0
\end{aligned}
$$

so that $V \subseteq \operatorname{ker}\left(\widehat{\pi}^{\prime}\right)$ which implies that $A \otimes v \subseteq A \otimes \operatorname{ker}\left(\widehat{\pi}^{\prime}\right)$.

Take $u \in \operatorname{ker}\left(\widehat{\pi}^{\prime}\right)$. Then $\widehat{\pi}^{\prime}(u)=0$ so that

$$
\begin{aligned}
\widehat{\pi}\left(t^{m} \otimes u\right) & =t^{m} \otimes \widehat{\pi}^{\prime}(u) \\
& =t^{m} \otimes 0 \\
& =0
\end{aligned}
$$

so that $A \otimes \operatorname{ker}\left(\widehat{\pi}^{\prime}\right)=A \otimes V=\widehat{M}(\alpha, \gamma)$.

Using the map $\widehat{\pi}^{\prime}$, we will be able to show that all but one of the terms in the $W_{1}$-action of $e_{k}$ on $\sigma_{0}$ given in $(3.4 .2)$ will lie in a finite dimensional module of $\widehat{M}(\alpha, \gamma)$. Proposition 3.3.2 will then come in handy to prove what kind of function our cocycles could be.

Since $1 \otimes \widehat{\pi}^{\prime}(\sigma)=\widehat{\pi}\left(\sigma_{0}\right)=\varepsilon_{\gamma}=1 \otimes \varepsilon$, we see that $\widehat{\pi}^{\prime}(\sigma)=\varepsilon$ so that

$$
\begin{aligned}
\widehat{\pi}\left(e_{k} \sigma_{0}\right) & =t^{k} \otimes \widehat{\pi}^{\prime}\left(\sum_{i=1}^{\infty} \frac{k^{i}}{i !} \rho\left(z^{i} \frac{d}{d z}\right) \varepsilon\right) \\
& =t^{k} \otimes\left(\sum_{i=1}^{\infty} \frac{k^{i}}{i !} \rho\left(z^{i} \frac{d}{d z}\right) \widehat{\pi}^{\prime}(\varepsilon)\right) \\
& =t^{k} \otimes\left(\sum_{i=1}^{\infty} \frac{k^{i}}{i !} \rho\left(z^{i} \frac{d}{d z}\right) \varepsilon\right) \\
& =t^{k} \otimes(\beta k \varepsilon)
\end{aligned}
$$


By looking at the formula 3.4 .2 ,

$$
t^{k} \otimes k(\beta \varepsilon)=t^{k} \otimes\left(k \widehat{\pi}^{\prime}\left(u_{1}\right)+k^{2} \widehat{\pi}^{\prime}\left(u_{2}\right)+\cdots+k^{n} \widehat{\pi}^{\prime}\left(u_{n}\right)\right)
$$

so that $k^{2} \widehat{\pi}^{\prime}\left(u_{2}\right)+\cdots+k^{n} \widehat{\pi}^{\prime}\left(u_{n}\right)=0$ for all $k \in \mathbb{Z}$. Since $\left\{k^{2}, k^{3}, \ldots, k^{n}\right\}$ are linearly independent, this implies that $\widehat{\pi}^{\prime}\left(u_{i}\right)=0$ for $2 \leq i \leq n$. But then $\left\{u_{i} \mid 2 \leq i \leq n\right\} \subset \operatorname{ker}\left(\widehat{\pi}^{\prime}\right)$ so that $t^{k} \otimes u_{i} \in \widehat{M}(\alpha, \gamma)$ for $2 \leq i \leq n$.

Equation 3.4 .2$)$ is reduced to

$$
\begin{aligned}
t^{k} \otimes k(\beta \varepsilon) & =t^{k} \otimes\left(k \widehat{\pi}^{\prime}\left(u_{i}\right)\right) \\
t^{k} \otimes 0 & =t^{k} \otimes k\left(\widehat{\pi}^{\prime}\left(u_{i}\right)-\beta \varepsilon\right)
\end{aligned}
$$

We can conclude that $t^{k} \otimes k\left(\widehat{\pi}^{\prime}\left(u_{1}\right)-\beta \varepsilon\right)=t^{k} \otimes k \widehat{\pi}^{\prime}(\omega)=0$ for some $\omega \in \operatorname{ker}\left(\widehat{\pi}^{\prime}\right)$. Thus $t^{k} \otimes \omega \in \widehat{M}(\alpha, \gamma)$ and $\widehat{\pi}^{\prime}\left(u_{1}\right)=\beta \varepsilon+\widehat{\pi}^{\prime}(\omega)$.

Now,

$$
\widehat{\pi}\left(e_{k} \sigma_{0}\right)=t^{k} \otimes\left(k \beta \varepsilon+k \widehat{\pi}^{\prime}(\omega)+k^{2} \widehat{\pi}^{\prime}\left(u_{2}\right)+\cdots+k^{n} \widehat{\pi}^{\prime}\left(u_{n}\right)\right)
$$

Since $t^{k} \otimes\left(k \omega+k^{2} u_{2}+\cdots+k^{n} u_{n}\right) \subset \widetilde{M}_{k+\gamma}$ and are contained in $\widehat{M}(\alpha, \gamma)$, they are contained in $\widehat{M}(\alpha, \gamma)_{k+\gamma}$. As $\widehat{M}(\alpha, \gamma) \subset \operatorname{Hom}(A, T(\alpha, \gamma))$, we may apply Proposition 3.3 .2 to conclude that $t^{k} \otimes\left(k \omega+k^{2} u_{2}+\cdots+k^{n} u_{n}\right)$ can be written in the form $\widetilde{\tau}(k)$ where $\widetilde{\tau}(k)$ is a linear combination of $\left\{\theta_{k}^{(0)}, \ldots, \theta_{k}^{(N)}, \delta_{k}\right\}$. Notice that $\widetilde{\tau}_{k} \subseteq \widehat{M}(\alpha, \gamma)_{k+\gamma}$.

Finally, we can say something about the $W_{1}$-action on $\widehat{w}_{m}$.

$$
\begin{aligned}
e_{k} \widehat{w}_{m} & =e_{k} \pi\left(\sigma_{m}\right) \\
& =e_{k}\left(\sigma_{m}(1)\right)
\end{aligned}
$$




$$
\begin{aligned}
& =e_{k} \sigma_{0}\left(t^{m}\right) \\
& =\left(e_{k} \sigma_{0}\right)\left(t^{m}\right)+\sigma_{0}\left(e_{k} t^{m}\right) \\
& =t^{k} \otimes\left(k u_{1}+k^{2} u_{2}+\cdots+k^{n} u_{n}\right)\left(t^{m}\right)+m \widehat{w}_{k+m} \\
& =t^{k} \otimes k \beta \varepsilon\left(t^{m}\right)+\widetilde{\tau}(k)\left(t^{m}\right)+m \widehat{w}_{k+m} \\
& =(m+\beta k) \widehat{w}_{k+m}+\widetilde{\tau}(k)\left(t^{m}\right)
\end{aligned}
$$

Notice that as for all $k, m \in \mathbb{Z}$,

$$
\begin{aligned}
\widetilde{\tau}(k)\left(t^{m}\right) & =t^{k} \otimes\left(k \omega+k^{2} u_{2}+\cdots+k^{n} u_{n}\right)\left(t^{m}\right) \\
& =t^{k+m} \otimes\left(k \omega+k^{2} u_{2}+\cdots+k^{n} u_{n}\right)(1) \\
& =\pi\left(t^{m+k} \otimes\left(k \omega+k^{2} u_{2}+\cdots+k^{n} u_{n}\right)\right)
\end{aligned}
$$

so that $\widetilde{\tau}(k)\left(t^{m}\right) \in M_{k+m+\gamma}$. Also $\widetilde{\tau}(k)=\sum_{i=0}^{N} c_{i} \theta_{k}^{(i)}$ so that

$$
\begin{aligned}
\widetilde{\tau}(k)\left(t^{m}\right) & =\sum_{i=0}^{N} c_{i} \theta_{k+\gamma}^{(i)}\left(t^{m}\right) \\
& =\sum_{i=0}^{N} c_{i} \frac{(k+m+\gamma)^{i}}{i !} v_{m+k+\gamma} \\
& =\sum_{i=0}^{N} c_{i} \theta_{k+m+\gamma}^{(i)}\left(t^{0}\right) \\
& =\sum_{i=0}^{N} c_{i} \pi\left(\theta_{k+m+\gamma}\right)
\end{aligned}
$$

so that we may think of $\widetilde{\tau}(k)\left(t^{m}\right)$ as the image under $\pi$ of a polynomial $\widetilde{\tau}(k, m)$ in $k$ and $\theta_{m+k+\gamma}$ functions. Thus,

$$
\pi(\widetilde{\tau}(k, m))=\tau(k, m) v_{m+k+\gamma}
$$


where $\tau(k, m)$ is a polynomial in $k$ and $m$. We may shift $m \in \mathbb{Z}$ to $m \in \gamma+\mathbb{Z}$ to simplify our notation.

Now we apply the surjective map from $M$ to $T(\beta, \gamma)$ which we denote by $v \rightarrow \bar{v}$ for $v \in M, \bar{v} \in T(\beta, \gamma)$. Since $\widetilde{\tau}(k, m) \in \widehat{M}(\alpha, \gamma)$, using that $\pi(\widehat{M}(\alpha, \gamma)) \subset T(\alpha, \gamma)$ and $\pi(\widehat{T(\beta, \gamma)}) \subseteq T(\beta, \gamma)$ we obtain

$$
\begin{aligned}
\overline{e_{k} \widehat{w}_{m}} & =\overline{(m+\beta k) \widehat{w}_{m+k}}+\overline{\pi(\widetilde{\tau}(k, m))} \\
& =k \beta w_{k+m}+m w_{k+m}
\end{aligned}
$$

This shows that $\zeta(k, m)=0$, so that $e_{k} \widehat{w}_{m}=(m+\beta k) \widehat{w}_{k+m}+\tau(k, m) v_{k+m}$. We can identify $T(\beta, \gamma)$ in $\widetilde{M}$ by using basis vectors $\varepsilon_{m}=t^{m} \otimes \sigma$ so that $\varepsilon_{m}(1)=\widehat{w}_{m}$. Then we find that the $W_{1}$-action on $\widetilde{M}$ is given by

$$
e_{k} \varepsilon_{m}=(m+\beta k) \varepsilon_{k+m}+\widetilde{\tau}(k, m)
$$

where $\widetilde{\tau}(k, m)$ is in a submodule in $\operatorname{Hom}(A, T(\alpha, \gamma))$ of the form in Proposition 3.3.2. Thus when $\beta \neq 1, \widetilde{\tau}(k, m)$ is a polynomial in $k$ and $\theta_{k+m}$ with the possibility of a $\delta_{k+m}$-function component when $\alpha=0$.

This is enough to show that our cocycles are polynomial. By Proposition 3.3.2. we know that $\widetilde{\tau}(k)$ is contained in the submodule $\left\{\theta_{k}^{(0)}, \ldots, \theta_{k}^{(N)}, \delta_{n}\right\}$ for some $N$. Thus, $\widehat{M}(\alpha, \gamma)$ is contained in a module $\widehat{M}_{N}(\alpha, \gamma)$ where $\widehat{M}_{N}(\alpha, \gamma)$ is the submodule spanned by $\left\{\theta_{k}^{(0)}, \ldots, \theta_{k}^{(N)}, \delta_{n}\right\}$. As $\widehat{M}(\alpha, \gamma) \subseteq \widehat{M}_{N}(\alpha, \gamma)$, we may extend our module $\widetilde{M}$ to $\widetilde{M}_{N}$ which is given by the extension

$$
0 \rightarrow \widehat{M}_{N}(\alpha, \gamma) \rightarrow \widetilde{M}_{N} \rightarrow T(\beta, \gamma) \rightarrow 0
$$

This will be convenient in the next section when we consider $\widetilde{\tau}$ cocycles as we may 
assume that $\theta_{k}^{(i)}$ is contained in our submodule for each $i \in \mathbb{N}$. As $\widetilde{M} \subset \widetilde{M}_{N}$, then $\pi: \widetilde{M}_{N} \rightarrow M$ will be surjective for $\beta \neq 1$ and $(\alpha \neq 1$ or $\gamma \notin \mathbb{Z})$, which will allow us reclaim the cocycles from the original extension of $M$. Also, as $\theta_{0}^{(0)} \in \widetilde{M}_{N}$, $\theta_{0}^{(0)}\left(t^{0}\right)=v_{0}$ so that $\pi: \widetilde{M}_{N}(\alpha, \gamma) \rightarrow T(\alpha, \gamma)$ is surjective even when $\alpha=1$ and $\gamma=0$. 


\section{Chapter 4}

\section{Polynomial Cocycles}

From the previous section, we found that for $\alpha \neq 0, \beta \neq 1$, every 1-cocycle is a polynomial function so that we can assume $\tau(k, m)=\sum_{i=0}^{n} c_{i} k^{i} m^{n-i}$ for some $c_{i} \in \mathbb{C}$. First, we will work with $\widetilde{\tau}(k, m)$ and then consider the homomorphism $\pi: \widetilde{M}_{N} \rightarrow M$ to obtain the functions $\tau(k, m)$. In this section, we will derive some general results about the coefficients $c_{i}$ of $\tau(k, m)$, then we will look at each case of $n \in \mathbb{N}_{+}$separately to find a classification of all polynomial 1-cocycles.

\subsection{General results for polynomial cocycles}

The $W_{1}$-action on $\varepsilon_{m}$ is given by

$$
e_{k} \cdot \varepsilon_{m}=(m+\beta k) \varepsilon_{k+m}+\sum_{i=0}^{n} \sum_{\ell=0}^{s} c_{i} k^{\ell} \theta_{k+m}^{(i)}
$$

where $k, n, s \in \mathbb{Z}$ and $m \in \gamma+\mathbb{Z}$. The space of polynomials $\mathbb{C}[k, m]$ admits a $\mathbb{Z}$-grading, where homogeneous elements of degree $n$ consist of monomials whose powers of $k$ and $m$ sum to $n$. Using (3.1.2), we can see that each homogeneous component of the polynomial $\tau(k, m)$ must independently satisfy the cocycle con- 
dition. Similarly, this is true for the coboundary condition given in Chapter 3.

For the cocycle $\widetilde{\tau}(k, m)$, we may introduce an analogous idea of a homogeneous element. A homogeneous element of degree $n$ will consist of monomials $k^{\ell} \theta_{k+m}^{(i)}$ for which $\ell+i=n$. In this way, we obtain a $\mathbb{Z}$-grading on the cohomology space. Again, each homogeneous component of $\widetilde{\tau}(k, m)$ will independently satisfy 3.1 .2 and (4.1.7), so that it is enough to consider homogeneous cocycles

$$
e_{k} \cdot \varepsilon_{m}=(m+\beta k) \varepsilon_{m+k}+\sum_{i=0}^{n} c_{i} k^{n-i} \theta_{m+k}^{(i)}
$$

By Theorem 3.4.1,

$$
\begin{gathered}
e_{k} \cdot \varepsilon_{m}=m \varepsilon_{m+k}+\sum_{i=1}^{\infty} \frac{k^{i}}{i !}\left(\rho\left(z^{i} \frac{d}{d z}\right) \varepsilon\right)_{(m+k)} \\
e_{k} \cdot \theta_{m}^{(p)}=m \theta_{m+k}^{(p)}+\sum_{i=1}^{\infty} \frac{k^{i}}{i !}\left(\rho\left(z^{i} \frac{d}{d z}\right) \theta^{(p)}\right)_{(m+k)}
\end{gathered}
$$

Remark 4.1.1. By comparing powers of $k$ in 4.1.1) and 4.1.2), $c_{n}=0$ for every $m \in \gamma+\mathbb{Z}$

Lemma 4.1.2. The $W_{1}$-action on $\theta_{j}^{(p)}$ is given by

$$
\begin{aligned}
e_{k} \cdot \theta_{j}^{(p)}= & \sum_{i=0}^{p-1} \frac{(-1)^{p-i}}{(p+1-i) !}((p+1-i) \alpha-(p+1)) k^{p+1-i} \theta_{j+k}^{(i)} \\
& +(j+(\alpha-p) k) \theta_{j+k}^{(p)}
\end{aligned}
$$

Proof. Using the $W_{1}$-action defined on $\operatorname{Hom}(A, T(\alpha, \gamma))$ in Chapter 2,

$$
\begin{aligned}
\left(e_{k} \theta_{j}^{(p)}\right)\left(t^{m}\right) & =e_{k}\left(\theta_{j}^{(p)}\left(t^{m}\right)\right)-\theta_{j}^{(p)}\left(e_{k} t^{m}\right) \\
& =\frac{(m+j)^{p}}{p !} e_{k} \cdot v_{m+j}-m \theta_{j}^{(p)}\left(t^{m+k}\right) \\
& =\frac{(m+j)^{p}}{p !}(m+j+\alpha k) v_{m+j+k}-m \frac{(m+j+k)^{p}}{p !} v_{m+j+k}
\end{aligned}
$$


Then, by applying (4.1.4 to $t^{m}$, we would like to show that this expression is equal to $\frac{(m+j)^{p}}{p !}(m+j+\alpha k) v_{m+j+k}-m \frac{(m+j+k)^{p}}{p !} v_{m+j+k}$. Let $D$ denote the difference of these two expressions. Then

$$
\begin{aligned}
& D=\sum_{i=0}^{p-1} \frac{(-1)^{p-i}}{(p+1-i) !}((p+1-i) \alpha-(p+1)) k^{p+1-i} \frac{(m+j+k)^{i}}{i !} \\
& +(j+(\alpha-p) k) \frac{(m+j+k)^{p}}{p !}-\frac{(m+j)^{p}}{p !}(m+j+\alpha k)+m \frac{(m+j+k)^{p}}{p !} \\
& p ! D=\sum_{i=0}^{p-1}(-1)^{p-i}\left(\left(\begin{array}{c}
p \\
i
\end{array}\right) \alpha-\left(\begin{array}{c}
p+1 \\
i
\end{array}\right)\right) k^{p+1-i}(m+j+k)^{i} \\
& +(j+m+(\alpha-p) k)(m+j+k)^{p}-(m+j)^{p}(m+j+\alpha k) \\
& p ! D=\sum_{i=0}^{p}(-1)^{p-i}\left(\left(\begin{array}{c}
p \\
i
\end{array}\right) \alpha-\left(\begin{array}{c}
p+1 \\
i
\end{array}\right)\right) k^{p+1-i}(m+j+k)^{i} \\
& -(\alpha-(p+1)) k(j+k+m)^{p}+(j+m+(\alpha-p) k)(m+j+k)^{p} \\
& -(m+j)^{p}(m+j+\alpha k) \\
& p ! D=k \sum_{i=0}^{p}(-k)^{p-i}\left(\begin{array}{l}
p \\
i
\end{array}\right) \alpha(-k)^{p-i}(m+j+k)^{i}-(m+j)^{p}(m+j+\alpha k) \\
& +\sum_{i=0}^{p}\left(\begin{array}{c}
p+1 \\
i
\end{array}\right)(-k)^{p+1-i}(m+j+k)^{i}+(j+k+m)^{p+1} \\
& p ! D=k(m+j)^{p}-(m+j)^{p}(m+j+\alpha k)+\sum_{i=0}^{p+1}\left(\begin{array}{c}
p+1 \\
i
\end{array}\right)(-k)^{p+1-i}(m+j+k)^{i} \\
& -(m+j+k)^{p+1}+(j+k+m)^{p+1} \\
& p ! D=(m+j)^{p+1}-(m+j+k)^{p+1}+(j+k+m)^{p+1}-(m+j)^{p+1} \\
& p ! D=0
\end{aligned}
$$

Therefore, these expressions are equal.

Recall that a trivial cocycle is a cocycle in the equivalence class of the zero function. In Chapter 3, we derived that a 1-coboundary is of the form $-\left(e_{k} \varphi\right)\left(w_{i}\right)$ 
for $\varphi\left(w_{i}\right)=g(i) v_{i}, w_{i} \in M_{2}, v_{i} \in M_{1}$. For $\widetilde{\tau}(k, m), \varphi \in \operatorname{Hom}\left(T(\beta, \gamma), \widehat{M}_{N}(\alpha, \gamma)\right)$ such that $\varphi\left(\varepsilon_{i}\right)=g(i) \theta_{i}^{(p)}$, so that

$$
\begin{aligned}
\tilde{\tau}(k, m)= & \left(e_{k} \varphi\right)\left(\varepsilon_{m}\right) \\
= & -e_{k} \cdot \varphi\left(\varepsilon_{m}\right)+\varphi\left(e_{k} \cdot \varepsilon_{m}\right) \\
= & -g(m) e_{k} \cdot \theta_{m}^{(p)}+(m+\beta k) \varphi\left(\varepsilon_{m+k}\right) \\
= & (m+\beta k) g(m+k) \theta_{m+k}^{(p)}-g(m) e_{k} \cdot \theta_{m}^{(p)} \\
= & m(g(m+k)-g(m)) \theta_{m+k}^{(p)}+(\beta k g(m+k)-(\alpha-p) k g(m)) \theta_{m+k}^{(p)} \\
& -\sum_{i=0}^{p-1} \frac{(-1)^{p-i}}{(p+1-i) !}((p+1-i) \alpha-(p+1)) g(m) k^{p+1-i} \theta_{m+k}^{(i)}
\end{aligned}
$$

Generally, $g(m)$ may have few restrictions. In particular though, we would like to find when our polynomial cocycles are coboundaries. Thus, we would like to find coboundaries that are homogeneous polynomials in $k$ and $\theta_{m+k}^{(i)}$. By comparing the coefficient of $\theta_{m+k}^{(0)}$ for $\alpha \neq 1$ on both sides of the above equality, this coefficient must be a polynomial in $k$ and independent of $m$. Thus for $p \geq 1, g(m)$ must be a constant. If $\alpha=1$ and $p \geq 2$, we may consider the coefficient of $\theta_{m+k}^{(1)}$ to come to the same conclusion.

We still need to consider the case of $p=0$ and $(p=1$ and $\alpha=1)$. The coefficient of $\theta^{(p)}$ is given by

$$
(m+\beta k) g(m+k)-(m+(\alpha-p) k) g(m)
$$

This must be a polynomial in $k$. If we set $m=\gamma$ then

$$
(\gamma+\beta k) g(\gamma+k)-(\gamma+(\alpha-p) k) g(\gamma)
$$


As $g(\gamma)$ is a constant, then $(\gamma+\beta k) g(\gamma+k)$ must be a polynomial in $k$. Then $g(x)=\frac{q_{1}(x)}{(\beta x-(1-\beta) \gamma)}$ for some polynomial $q_{1}(x)$. If we set $m=\gamma+1$, then

$$
(\gamma+1+\beta k) g(\gamma+1+k)-(\gamma+1+(\alpha-p) k) g(\gamma+1)
$$

As $g(\gamma+1)$ is a constant, then the first term must be polynomial in $k$. Then $g(x)=\frac{q_{2}(x)}{(\beta x+(1-\beta)(\gamma+1))}$ for some polynomial in $k$. Thus,

$$
\frac{q_{1}(x)}{(\beta x-(1-\beta) \gamma)}=\frac{q_{2}(x)}{(\beta x+(1-\beta)(\gamma+1))}
$$

so that either $(\beta x-(1-\beta) \gamma)=(\beta x+(1-\beta)(\gamma+1))$ otherwise $g(x)$ is a polynomial function in $x$. Let us look at the first case a bit further.

If these two factors are equal, then $\beta=1$ and for some polynomial $q(x)$ and some constant $c$,

$$
g(x)= \begin{cases}\frac{q(x)}{x}, & x \neq 0 \\ c, & x=0\end{cases}
$$

so that

$$
(\gamma+k) g(\gamma+k)-(\gamma+(\alpha-p) k) g(\gamma)=q(\gamma+k)-\frac{(\gamma+(\alpha-p) k)}{\gamma} q(\gamma)
$$

and thus $\alpha=p$ to obtain a polynomial coboundary. Notice for $p=1, \alpha=1$ this is already true and for $p=0$, this requires that $\alpha=0$ for a non-polynomial function $g(x)$. This reduces 4.1.5 to

$$
(m+k) g(m+k)-m g(m)=q(m+k)-q(m)+\delta_{m, 0} q(m)-\delta_{m+k, 0} q(m+k)
$$

for $(\alpha=0, p=0)$ and $(\alpha=1, p=1)$ so that 4.1 .5$)$ is a polynomial function in $m$ 
and $k$ with a possible difference of delta functions.

Here, we conclude that there are two different types of non-trivial coboundaries that arise:

1. When $g(m)$ is a polynomial function in $m$, it must be a constant. This admits coboundaries that are homogeneous polynomials in $k$ and $\theta$.

2. When both $\alpha=0$ and $\beta=1$, the coboundaries that arise are given by

$$
q(m+k)-q(m)+\delta_{m, 0} q(m)-\delta_{m+k, 0} q(m+k)
$$

which admits the delta-function coboundary

$$
\delta_{m, 0}-\delta_{m+k, 0}
$$

Therefore for strictly homogeneous polynomial coboundaries, $\widetilde{\tau} \sim 0$ if there exists $h \in \mathbb{C}$ such that

$$
\begin{aligned}
\tau(k, j)= & h \sum_{i=0}^{p-1} \frac{(-1)^{p-i}}{(p+1-i) !}((p+1-i) \alpha-(p+1)) k^{p+1-i} \theta_{j+k}^{(i)} \\
& +h k(\alpha-\beta-p) \theta_{j+k}^{(p)}
\end{aligned}
$$

We can obtain some more information about the representation $\rho$, which will be a useful our calculations. From (4.1.1) - 4.1.4, we can derive the formulae:

$$
\begin{aligned}
\rho\left(z \frac{d}{d z}\right) \theta^{(p)} & =(\alpha-p) \theta^{(p)}, \\
\rho\left(z^{i} \frac{d}{d z}\right) \theta^{(p)} & =(-1)^{i-1}(i \alpha-(p+1)) \theta^{(p+1-i)}, i \geq 2 \\
\rho\left(z \frac{d}{d z}\right) \varepsilon & =\beta \varepsilon+c_{n-1} \theta^{(n-1)}, \\
\rho\left(z^{i} \frac{d}{d z}\right) \varepsilon & =i ! c_{n-i} \theta^{(n-i)}, i \geq 2
\end{aligned}
$$


Also, since $\rho$ is a representation,

$$
\left[\rho\left(z^{i} \frac{d}{d z}\right), \rho\left(z^{j} \frac{d}{d z}\right)\right] u=(j-i) \rho\left(z^{i+j-1} \frac{d}{d z}\right) u
$$

The next two results will determine the conditions on the coefficients $c_{i}$ using (4.1.12).

Lemma 4.1.3. For all $n \in \mathbb{N}_{+}$, and for $i, j \in \mathbb{Z}_{+}$,

1. For $i \geq 2$,

$$
(\alpha-\beta-(n-1)) c_{n-i}=(-1)^{i-1}(i \alpha-n) c_{n-1}
$$

2. For $i, j \geq 2, i+j \leq n-1$, and $i \neq j$

$$
\begin{aligned}
(i+j-1) !(j-i) c_{n-i-j+1}= & j !(-1)^{i-1}(i \alpha-(n-j+1)) c_{n-j} \\
& -i !(-1)^{j-1}(j \alpha-(n-i+1)) c_{n-i}
\end{aligned}
$$

Proof. For part (1), set $j=1, u=\varepsilon$ so by 4.1.12,

$$
\begin{aligned}
\text { LHS }= & \rho\left(z^{i} \frac{d}{d z}\right) \rho\left(z \frac{d}{d z}\right) \varepsilon-\rho\left(z \frac{d}{d z}\right) \rho\left(z^{i} \frac{d}{d z}\right) \varepsilon \\
= & \rho\left(z^{i} \frac{d}{d z}\right)\left(\beta \varepsilon+c_{n-1} \theta^{(n-1)}\right)-\rho\left(z \frac{d}{d z}\right)\left(i ! c_{n-i} \theta^{(n-i)}\right) \\
= & i !(-1)^{i-1}(i \alpha-n) c_{n-1} \theta^{(n-i)}-i !(\alpha-(n-i)) c_{n-i} \theta^{(n-i)} \\
& +\beta i ! c_{n-i} \theta^{(n-i)} \\
R H S= & (1-i) i ! c_{n-i} \theta^{(n-i)} \\
\Longrightarrow & (\alpha-\beta-(n-1)) c_{n-i}=(-1)^{i-1}(i \alpha-n) c_{n-1}
\end{aligned}
$$


For part (2), by using for $i, j \geq 2$ in 4.1.12,

$$
\begin{aligned}
\text { LHS }= & \rho\left(z^{i} \frac{d}{d z}\right) \rho\left(z^{j} \frac{d}{d z}\right) \varepsilon-\rho\left(z^{j} \frac{d}{d z}\right) \rho\left(z^{i} \frac{d}{d z}\right) \varepsilon \\
= & \rho\left(z^{i} \frac{d}{d z}\right)\left(j ! c_{n-j} \theta^{(n-j)}\right)-\rho\left(z^{j} \frac{d}{d z}\right)\left(i ! c_{n-i} \theta^{(n-i)}\right) \\
= & j !(-1)^{i-1}(i \alpha-(n-j+1)) c_{n-j} \theta^{(n-i+1-j)} \\
R H S= & (i+j-1) !(j-i) c_{n-i-j+1} \\
\Longrightarrow & (i+j-1) !(j-i) c_{n-i-j+1}=(-1)^{i-1} j !(i \alpha-(n-j+1)) c_{n-j} \\
& -(-1)^{j-1} i !(j \alpha-(n-i+1)) c_{n-i}
\end{aligned}
$$

Theorem 4.1.4. For non-trivial 1-cocycles with $n \geq 3, c_{n-1}=0$ and $\alpha-\beta=n-1$.

Proof. Suppose $n \geq 3$ and suppose $c_{n-1}$ is non-zero. Then by (4.1.13),

$$
i !(\alpha-\beta-(n-1)) c_{n-i} \neq 0
$$

for all $i$, and this $(\alpha-\beta-(n-1))$ is necessarily non-zero. Thus

$$
c_{n-i}=\frac{(-1)^{i-1}(i \alpha-n)}{i !(\alpha-\beta-(n-1))} c_{n-1}
$$

So that the $W_{1}$ action on $\varepsilon_{m}$ is

$$
\begin{aligned}
e_{k} \cdot \varepsilon_{m} & =(m+\beta k) \varepsilon_{m+k}+k c_{n-1} \theta_{m}^{(n-1)}+\sum_{i=0}^{n-2} c_{i} k^{n-i} \theta_{m}^{(i)} \\
& =(m+\beta k) \varepsilon_{m+k}+k c_{n-1} \theta_{m}^{(n-1)}+\sum_{i=2}^{n} c_{n-i} k^{i} \theta_{m}^{(n-i)}
\end{aligned}
$$




$$
\begin{aligned}
= & (m+\beta k) \varepsilon_{m+k}+k c_{n-1} \theta_{m}^{(n-1)}+\sum_{i=2}^{n} \frac{(-1)^{i-1}(i \alpha-n)}{i !(\alpha-\beta-(n-1))} c_{n-1} k^{i} \theta_{m}^{(n-i)} \\
= & (m+\beta k) \varepsilon_{m+k}+k c_{n-1} \theta_{m}^{(n-1)} \\
& +\frac{c_{n-1}}{(\alpha-\beta-(n-1))} \sum_{i=0}^{n-2} \frac{(-1)^{n-i-1}((n-i) \alpha-n)}{(n-i) !} k^{n-i} \theta_{m}^{(i)}
\end{aligned}
$$

Take $h=\frac{c_{n-1}}{\alpha-\beta-(n-1)} \in \mathbb{C}$. Then this 1-cocycle is equivalent to the trivial 1-cocycle. For non-trivial cocycles, $c_{n-1}$ must be zero, so $\alpha-\beta-(n-1)$ must be zero as well if $\widetilde{\tau}$ is non-trivial.

From this section, we obtain two crucial conditions for non-trivial 1-cocycles of degree greater than two: $c_{n-1}=0$, and $\alpha-\beta=n-1$.

\subsection{Cases when $n \leq 4$}

When $n \leq 4$, there does not exist positive integers $i$ and $j$ satisfying $i \geq 2, j \geq 2$ and $i \neq j$ such that $n-i-j+1$ is still positive. This implies that Part (2) of Lemma 4.1 .3 cannot be used. Instead, each case must be carefully looked at.

\subsubsection{Cocycles of degree 1}

When $n=1$, we cannot use Part (1) of Lemma 4.1.3, since there does not exist $i \geq 2$ such that $n-2 \geq 0$. Equality (4.1.1) does give us the possible form of the $W_{1}$ action:

$$
e_{k} \cdot \varepsilon_{m}=(m+\beta k) \varepsilon_{m+k}+c_{0} k \theta_{m+k}^{(0)}
$$

From this equation, we can derive the representation $\rho$ :

$$
\rho\left(z \frac{d}{d z}\right)=\left[\begin{array}{cc}
\alpha & c_{0} \\
0 & \beta
\end{array}\right], \quad \rho\left(z^{i} \frac{d}{d z}\right)=0, \text { for } i \geq 2
$$


For valid cocycles, $\rho$ must satisfy the condition 4.1 .12 but as $\left[\rho\left(z \frac{d}{d z}\right), \rho\left(z \frac{d}{d z}\right)\right]=$ 0 , this tells us that there are no restrictions on $c_{0}$ as any value will give a valid representation.

For trivial cocycles, by $4.1 .7 h \in \mathbb{C}$ must exist such that

$$
c_{0} k \theta_{m+k}^{(0)}=h k(\alpha-\beta) \theta_{m+k}^{(0)}
$$

If $\alpha \neq \beta$, then $h=\frac{c_{0}}{\alpha-\beta}$. When $\alpha=\beta$, the equivalence relationship is simply zero, so only cocycles strictly equal can be equivalent. Thus for any non-zero $c_{0}$, the cocycle is non-trivial, which gives an equivalence class of $k \theta^{(0)}$.

\subsubsection{Cocycles of degree 2}

When $n=2$, Part (1) of Lemma 4.1.3 applies and we obtain the single equation for $i=2$ :

$$
i !(\alpha-\beta-(n-1)) c_{n-i}=(-1)^{i-1}(i \alpha-n) c_{n-1}
$$

By the proof of Theorem 4.1.4, if $\alpha-\beta-(n-1) \neq 0$, this cocycle will be trivial. Thus, $\alpha-\beta=1$ and we get two cases when the above equation is equal to zero: $c_{n-1}=0$, or $(i \alpha-n)=2 \alpha-2=0$, which is simply the case of $\alpha=1$.

Case of $c_{n-1}=0$

When $c_{n-1}=c_{1}=0$, by 4.1.1,

$$
e_{k} \cdot \varepsilon_{m}=(m+\beta k) \varepsilon_{m+k}+c_{0} k^{2} \theta_{m+k}^{(0)}
$$


We can explicitly write out the representation $\rho$ :

$$
\begin{aligned}
\rho\left(z \frac{d}{d z}\right) & =\left[\begin{array}{ccr}
\alpha & 0 & 0 \\
0 & \alpha-1 & 0 \\
0 & 0 & \beta
\end{array}\right], \quad \rho\left(z^{2} \frac{d}{d z}\right)=\left[\begin{array}{ccc}
0 & -2 \alpha+2 & 2 c_{0} \\
0 & 0 & 0 \\
0 & 0 & 0
\end{array}\right] \\
\rho\left(z^{i} \frac{d}{d z}\right) & =0, \text { for } i \geq 3 .
\end{aligned}
$$

Since $\rho$ is a representation, 4.1.12 holds for all positive integers $i$ and $j$. When $i=1, j=2$ we obtain that the following must hold:

$$
\left[\begin{array}{ccc}
0 & -2 \alpha(\alpha-1) & 2 \alpha c_{0} \\
0 & 0 & 0 \\
0 & 0 & 0
\end{array}\right]-\left[\begin{array}{ccc}
0 & -2(\alpha-1)^{2} & 2 \beta c_{0} \\
0 & 0 & 0 \\
0 & 0 & 0
\end{array}\right]=\left[\begin{array}{ccc}
0 & -2 \alpha+2 & 2 c_{0} \\
0 & 0 & 0 \\
0 & 0 & 0
\end{array}\right]
$$

This is true for every $\alpha, \beta, c_{0}$ such that $\alpha-\beta=1$. The trivial cocycle is given by 4.1.7) when $p=n-1=1$, or

$$
-(\alpha-1) k^{2} \theta_{m+k}^{(0)}
$$

so if $\alpha \neq 1$, every cocycle is trivial by setting $h=\frac{c_{0}}{\alpha-1}$. Therefore, from this case we obtain an equivalence class of $k^{2} \theta^{(0)}$ for $\alpha=1$ and $\beta=0$.

Case of $\alpha=1$

When $\alpha=1$, then $\beta=0$. By 4.1.1,

$$
e_{k} \cdot \varepsilon_{m}=m \varepsilon_{m+k}+c_{0} k^{2} \theta_{m+k}^{(0)}+c_{1} k \theta_{m+k}^{(1)}
$$


We again write out the representation $\rho$ :

$$
\begin{array}{ll}
\rho\left(z \frac{d}{d z}\right)=\left[\begin{array}{ccc}
1 & 0 & 0 \\
0 & 0 & c_{1} \\
0 & 0 & 0
\end{array}\right], & \rho\left(z^{2} \frac{d}{d z}\right)=\left[\begin{array}{ccc}
0 & 0 & 2 c_{0} \\
0 & 0 & 0 \\
0 & 0 & 0
\end{array}\right] \\
\rho\left(z^{i} \frac{d}{d z}\right) & =0, \text { for } i \geq 3 .
\end{array}
$$

Since $\rho$ is a representation, 4.1.12 holds for all positive integers $i$ and $j$. In particular, when $i=1, j=2$,

$$
\left[\begin{array}{ccc}
0 & 0 & 2 \alpha c_{0} \\
0 & 0 & 0 \\
0 & 0 & 0
\end{array}\right]-\left[\begin{array}{ccc}
0 & 0 & 0 \\
0 & 0 & 0 \\
0 & 0 & 0
\end{array}\right]=\left[\begin{array}{ccc}
0 & 0 & 2 c_{0} \\
0 & 0 & 0 \\
0 & 0 & 0
\end{array}\right]
$$

so that there are no restrictions on $c_{0}$ of $c_{1}$. The trivial cocycle is given by (4.2.1), which is exactly zero when $\alpha=1$. This gives us an equivalence class of $k^{2} \theta^{(0)}+$ $k \theta^{(1)}$, which can be reduced to $k \theta^{(1)}$ for $\alpha=1$ and $\beta=0$ by using the previous case.

\subsubsection{Cocycles of degree 3}

For the case of $n=3$, we can now make use of Theorem 4.1.4. For non-trivial cocycles, $\alpha-\beta=2$, and $c_{2}=0$. Then, by 4.1.1,

$$
e_{k} \cdot \varepsilon_{m}=(m+(\alpha-2) k) \varepsilon_{m+k}+c_{0} k^{3} \theta_{m+k}^{(0)}+c_{1} k^{2} \theta_{m+k}^{(1)}
$$


We explicitly write out the representation $\rho$ :

$$
\begin{aligned}
\rho\left(z \frac{d}{d z}\right) & =\left[\begin{array}{cccc}
\alpha & 0 & 0 & 0 \\
0 & \alpha-1 & 0 & 0 \\
0 & 0 & \alpha-2 & 0 \\
0 & 0 & 0 & \alpha-2
\end{array}\right] \\
\rho\left(z^{2} \frac{d}{d z}\right) & =\left[\begin{array}{llcc}
0 & -2 \alpha+2 & 0 & 0 \\
0 & 0 & -2 \alpha+3 & 2 c_{1} \\
0 & 0 & 0 & 0 \\
0 & 0 & 0 & 0
\end{array}\right] \\
\rho\left(z^{3} \frac{d}{d z}\right) & =\left[\begin{array}{lllc}
0 & 0 & 3 \alpha-3 & 6 c_{0} \\
0 & 0 & 0 & 0 \\
0 & 0 & 0 & 0 \\
0 & 0 & 0 & 0
\end{array}\right] \\
\rho\left(z^{i} \frac{d}{d z}\right) & =0, \text { for } i \geq 4 .
\end{aligned}
$$

Since $\rho$ is a representation, 4.1.12 holds for all positive integers $i$ and $j$. The only non-trivial equations will be obtained from $i=1, j=2$ and $i=1, j=3$. For any $c_{0}$ and $c_{1}, 4.1 .12$ will hold so that any values of $c_{0}$ and $c_{1}$ will give valid cocycles. All we need to do is find an element in the 1-dimensional non-trivial solution space to find the equivalence class of non-trivial cocycles. The trivial cocycle is given by (4.1.7) and is as follows:

$$
(\alpha-1) k^{3} \theta_{k+m}^{(0)}-(2 \alpha-3) k^{2} \theta_{k+m}^{(1)}
$$

For $c_{0}=1$, and $c_{2}=-2$, the cocycle is not trivial. Thus we obtain an equivalence class of $k^{3} \theta^{(0)}-2 k^{2} \theta^{(1)}$. 


\subsubsection{Cocycles of degree 4}

The case of $n=4$ is very similar to the previous case. For non-trivial cocycles, $\alpha-\beta=3$, and $c_{3}=0$. Then by (4.1.1),

$$
e_{k} \cdot \varepsilon_{m}=(m+(\alpha-3) k) \varepsilon_{m+k}+c_{0} k^{4} \theta_{m+k}^{(0)}+c_{1} k^{3} \theta_{m+k}^{(1)}+c_{2} k^{2} \theta_{m+k}^{(2)}
$$

The matrix form of the representation $\rho$ can be given by:

$$
\begin{aligned}
\rho\left(z \frac{d}{d z}\right) & =\left[\begin{array}{ccccc}
\alpha & 0 & 0 & 0 & 0 \\
0 & \alpha-1 & 0 & 0 & 0 \\
0 & 0 & \alpha-2 & 0 & 0 \\
0 & 0 & 0 & \alpha-3 & 0 \\
0 & 0 & 0 & 0 & \alpha-3
\end{array}\right] \\
\rho\left(z^{2} \frac{d}{d z}\right) & =\left[\begin{array}{lllccc}
0 & -2 \alpha+2 & 0 & 0 & 0 \\
0 & 0 & -2 \alpha+3 & 0 & 0 \\
0 & 0 & 0 & -2 \alpha+4 & 2 c_{2} \\
0 & 0 & 0 & 0 & 0 \\
0 & 0 & 0 & 0 & 0
\end{array}\right] \\
\rho\left(z^{3} \frac{d}{d z}\right) & =\left[\begin{array}{lllll}
0 & 0 & 3 \alpha-3 & 0 & 0 \\
0 & 0 & 0 & 3 \alpha-4 & 6 c_{1} \\
0 & 0 & 0 & 0 & 0 \\
0 & 0 & 0 & 0 & 0 \\
0 & 0 & 0 & 0 & 0
\end{array}\right]
\end{aligned}
$$




$$
\begin{aligned}
& \rho\left(z^{4} \frac{d}{d z}\right)=\left[\begin{array}{ccccc}
0 & 0 & 0 & -4 \alpha+4 & 24 c_{0} \\
0 & 0 & 0 & 0 & 0 \\
0 & 0 & 0 & 0 & 0 \\
0 & 0 & 0 & 0 & 0 \\
0 & 0 & 0 & 0 & 0
\end{array}\right] \\
& \rho\left(z^{i} \frac{d}{d z}\right)=0, \text { for } i \geq 5 .
\end{aligned}
$$

which imply the conditions:

$$
\begin{aligned}
& \text { For } i=1, j=2: 2(\alpha-\beta-3) c_{2}=0 \\
& \text { For } i=1, j=3: 6(\alpha-\beta-3) c_{1}=0 \\
& \text { For } i=1, j=4: 24(\alpha-\beta-3) c_{0}=0 \\
& \text { For } i=2, j=3: 12(\alpha-1) c_{1}+6(\alpha-1) c_{2}+24 c_{0}=0
\end{aligned}
$$

As $\alpha-\beta=3$, the first three conditions are trivial and by solving the last equation, we obtain that cocycles are of the form

$$
-\frac{(\alpha-1)}{4}\left(2 c_{1}+c_{2}\right) k^{4} \theta_{m+k}^{(0)}+c_{1} k^{3} \theta_{m+k}^{(1)}+c_{2} k^{2} \theta_{m+k}^{(2)}
$$

The 1-dimensional trivial solution space is given by (4.1.7) for $p=n-1=2$, or by the equivalence class of

$$
(\alpha-1) k^{4} \theta_{m+k}^{(0)}-(3 \alpha-4) k^{3} \theta_{m+k}^{(1)}+6(\alpha-2) k^{2} \theta_{m+k}^{(2)}
$$

Then the 1-dimensional non-trivial solution space is spanned by the cocycle where $2 c_{1}=-c_{2}$. Thus, we obtain the equivalence class of $k^{3} \theta^{(1)}-2 k^{2} \theta^{(2)}$. 


\subsection{Cases when $n \geq 5$}

As soon as $n \geq 5$, Part (2) of Lemma 4.1 .3 can be used. If we set $i=2$, then

$$
(j+1) !(2-j) c_{n-j-1}-2(-1)^{j-1}(j \alpha-n+1) c_{n-2}-j !(2 \alpha-n+j-1) c_{n-j}=0
$$

for $j=3,4, \ldots, n-2$ so that we will obtain $n-3$ formulas for appropriately large $n$. If we set $i=3$,

$$
(j+2) !(3-j) c_{n-j-2}-6(-1)^{j-1}(j \alpha-n+2) c_{n-3}+j !(3 \alpha-n+j-1) c_{n-j}=0
$$

for $j=4,5, \ldots, n-2$ so that we obtain $n-5$ formulas for appropriately large $n$. From this, we obtain the following coefficient matrix, where each column $j$ corresponds to the coefficient $c_{j-1}$.

$$
\left[\begin{array}{cccccccccc}
0 & 0 & 0 & \cdots & 0 & 0 & 0 & a_{1, n-4} & a_{1, n-3} & a_{1, n-2} \\
0 & 0 & 0 & \cdots & 0 & 0 & a_{2, n-5} & a_{2, n-4} & 0 & a_{2, n-2} \\
0 & 0 & 0 & \cdots & 0 & a_{3, n-6} & a_{3, n-5} & 0 & 0 & a_{3, n-2} \\
\vdots & \vdots & \vdots & & \vdots & \vdots & \vdots & \vdots & \vdots & \vdots \\
0 & a_{n-4,1} & a_{n-4,2} & \cdots & 0 & 0 & 0 & 0 & 0 & a_{n-4, n-2} \\
a_{n-3,0} & a_{n-3,1} & 0 & \cdots & 0 & 0 & 0 & 0 & 0 & a_{n-3, n-2} \\
0 & 0 & 0 & \cdots & 0 & b_{1, n-6} & 0 & b_{1, n-4} & b_{1, n-3} & 0 \\
0 & 0 & 0 & \cdots & b_{2, n-7} & 0 & b_{2, n-5} & 0 & b_{2, n-3} & 0 \\
\vdots & \vdots & \vdots & & \vdots & \vdots & \vdots & \vdots & \vdots & \vdots \\
0 & 0 & b_{n-7,2} & \cdots & 0 & 0 & 0 & 0 & b_{n-7, n-3} & 0 \\
0 & b_{n-6,1} & 0 & \cdots & 0 & 0 & 0 & 0 & b_{n-6, n-3} & 0 \\
b_{n-5,0} & 0 & b_{n-6,2} & \cdots & 0 & 0 & 0 & 0 & b_{n-5, n-3} & 0
\end{array}\right]
$$


where, for $1 \leq i \leq n-3$,

$$
\begin{aligned}
a_{i, n-2} & =2(-1)^{i+2}((i+2) \alpha-(n-1)) \\
a_{i, n-2-i} & =-(i+2) !(2 \alpha-(n-i-1)) \\
a_{i, n-3-i} & =-i(i+3) !
\end{aligned}
$$

and for $1 \leq i \leq n-5$,

$$
\begin{aligned}
b_{i, n-3} & =6(-1)^{i+3}((i+3) \alpha-(n-2)) \\
b_{i, n-3-i} & =(i+3) !(3 \alpha-(n-i-2)) \\
b_{i, n-5-i} & =-i(i+5) !
\end{aligned}
$$

This matrix encodes a system of $(n-3)+(n-5)=2 n-8$ equations in $n-1$ variables, our $n-1$ coefficients. We are guaranteed to always have a 1-dimensional trivial solution space, which means that the rank of our matrix is at most $n-2$. If there exists a non-trivial solution, then the rank would have to be less than or equal to $n-3$. 
To demonstrate this, we can further reduce this matrix to

$\left[\begin{array}{cccccccccc}0 & 0 & 0 & \cdots & 0 & 0 & 0 & a_{1, n-4} & a_{1, n-3} & a_{1, n-2} \\ 0 & 0 & 0 & \cdots & 0 & 0 & a_{2, n-5} & 0 & a_{2, n-3} & a_{2, n-2}^{\prime} \\ 0 & 0 & 0 & \cdots & 0 & a_{3, n-6} & 0 & 0 & a_{4, n-3} & a_{3, n-2}^{\prime} \\ \vdots & \vdots & \vdots & & \vdots & \vdots & \vdots & \vdots & \vdots & \vdots \\ 0 & a_{n-4,1} & 0 & \cdots & 0 & 0 & 0 & 0 & a_{n-4, n-3} & a_{n-4, n-2}^{\prime} \\ a_{n-3,0} & 0 & 0 & \cdots & 0 & 0 & 0 & 0 & a_{n-3, n-3} & a_{n-3, n-2}^{\prime} \\ 0 & 0 & 0 & \cdots & 0 & b_{1, n-6} & 0 & b_{1, n-4} & b_{1, n-3} & 0 \\ 0 & 0 & 0 & \cdots & b_{2, n-7} & 0 & b_{2, n-5} & 0 & b_{2, n-3} & 0 \\ \vdots & \vdots & \vdots & & \vdots & \vdots & \vdots & \vdots & \vdots & \vdots \\ 0 & 0 & b_{n-7,2} & \cdots & 0 & 0 & 0 & 0 & b_{n-7, n-3} & 0 \\ 0 & b_{n-6,1} & 0 & \cdots & 0 & 0 & 0 & 0 & b_{n-6, n-3} & 0 \\ b_{n-5,0} & 0 & b_{n-6,2} & \cdots & 0 & 0 & 0 & 0 & b_{n-5, n-3} & 0\end{array}\right]$

where, $2 \leq i \leq n-3$

$$
\begin{aligned}
a_{1, n-2}= & -2(3 \alpha-(n-1)) \\
a_{1, n-3}= & -6(2 \alpha-(n-2)) \\
a_{1, n-4}= & -24 \\
a_{i, n-2}^{\prime}= & \frac{2(-1)^{i}}{(i-1) !}\left(\left[\sum_{j=3}^{i+1}(j-3) !(j \alpha-(n-1)) \prod_{\ell=j}^{i+1}(2 \alpha-(n-\ell))\right]\right. \\
& +(i-1) !((i+2) \alpha-(n-1))) \\
a_{i, n-3}= & \frac{6(-1)^{i}}{(i-1) !} \prod_{j=1}^{i}(2 \alpha-(n-(j+1))) \\
a_{i, n-3-i}= & -i(i+3) !
\end{aligned}
$$


and for $1 \leq i \leq n-5$

$$
\begin{aligned}
b_{i, n-3} & =6(-1)^{i+3}((i+3) \alpha-(n-2)) \\
b_{i, n-3-i} & =(i+3) !(3 \alpha-(n-2-i)) \\
b_{i, n-5-i} & =-i(i+5) !
\end{aligned}
$$

Finally,

$$
\left[\begin{array}{cccccccccc}
0 & 0 & 0 & \cdots & 0 & 0 & 0 & a_{1, n-4} & a_{1, n-3} & a_{1, n-2} \\
0 & 0 & 0 & \cdots & 0 & 0 & a_{2, n-5} & 0 & a_{2, n-3} & a_{2, n-2}^{\prime} \\
0 & 0 & 0 & \cdots & 0 & a_{3, n-6} & 0 & 0 & a_{4, n-3} & a_{3, n-2}^{\prime} \\
\vdots & \vdots & \vdots & & \vdots & \vdots & \vdots & \vdots & \vdots & \vdots \\
0 & a_{n-4,1} & 0 & \cdots & 0 & 0 & 0 & 0 & a_{n-4, n-3} & a_{n-4, n-2}^{\prime} \\
a_{n-3,0} & 0 & 0 & \cdots & 0 & 0 & 0 & 0 & a_{n-3, n-3} & a_{n-3, n-2}^{\prime} \\
0 & 0 & 0 & \cdots & 0 & 0 & 0 & 0 & b_{1, n-3}^{\prime} & b_{1, n-2} \\
0 & 0 & 0 & \cdots & 0 & 0 & 0 & 0 & b_{2, n-3}^{\prime} & b_{2, n-2} \\
\vdots & \vdots & \vdots & & \vdots & \vdots & \vdots & \vdots & \vdots & \vdots \\
0 & 0 & 0 & \cdots & 0 & 0 & 0 & 0 & b_{n-7, n-3}^{\prime} & b_{n-7, n-2} \\
0 & 0 & 0 & \cdots & 0 & 0 & 0 & 0 & b_{n-6, n-3}^{\prime} & b_{n-6, n-2} \\
0 & 0 & 0 & \cdots & 0 & 0 & 0 & 0 & b_{n-5, n-3}^{\prime} & b_{n-5, n-2}
\end{array}\right]
$$

where, for $2 \leq i \leq n-3$,

$$
\begin{aligned}
& a_{1, n-2}=-2(3 \alpha-(n-1)) \\
& a_{1, n-3}=-6(2 \alpha-(n-2)) \\
& a_{1, n-4}=-24
\end{aligned}
$$




$$
\begin{aligned}
a_{i, n-2}^{\prime}= & \frac{2(-1)^{i}}{(i-1) !}\left(\left[\sum_{j=3}^{i+1}(j-3) !(j \alpha-(n-1)) \prod_{\ell=j}^{i+1}(2 \alpha-(n-\ell))\right]\right. \\
& +(i-1) !((i+2) \alpha-(n-1))) \\
a_{i, n-3}= & \frac{6(-1)^{i}}{(i-1) !} \prod_{j=1}^{i}(2 \alpha-(n-(j+1))) \\
a_{i, n-3-i}= & -i(i+3) !
\end{aligned}
$$

and for $1 \leq i \leq n-5$,

$$
\begin{aligned}
b_{i, n-2}= & \left(\frac{(3 \alpha-(n-2-i))}{i}\right) a_{i, n-2}^{\prime}-\left(\frac{i}{i+2}\right) a_{i+2, n-2}^{\prime} \\
b_{i, n-3}^{\prime}= & 6(-1)^{i+3}((i+3) \alpha-(n-2))-\left(\frac{i}{i+2}\right) a_{i+2, n-3} \\
& +\left(\frac{(3 \alpha-(n-2-i))}{i}\right) a_{i, n-3}
\end{aligned}
$$

which gives us the explicit formulae for $2 \leq i \leq n-5$

$$
\begin{aligned}
b_{1, n-2}= & \frac{1}{3}\left(-n^{3}+7 \alpha n^{2}+3 n^{2}-16 \alpha^{2} n-9 \alpha n-2 n+12 \alpha^{3}+6 \alpha\right) \\
b_{1, n-3}^{\prime}= & -n^{3}+6 \alpha n^{2}+3 n^{2}-12 \alpha^{2} n-6 \alpha n-2 n+8 \alpha^{3}+4 \alpha \\
b_{i, n-2}= & \frac{2(-1)^{i}}{i !}\left[\frac{i}{(i+1)(i+2)}\left(\sum_{j=1}^{i+3}(j-3) !(j \alpha-n+1) \prod_{\ell=j}^{i+3}(2 \alpha-(n-\ell))\right)\right. \\
& +(3 \alpha-n+2+i)\left(\sum_{j=1}^{i+1}(j-3) !(j \alpha-(n-1)) \prod_{\ell=j}^{i+1}(2 \alpha-(n-\ell))\right) \\
& \left.(i-1) !((i+2) \alpha-n+1)(3 \alpha-n+2+i)+\frac{i(i+1) !}{(i+2)(i+1)}((i+5) \alpha-n+1)\right] \\
b_{i, n-3}^{\prime} & =\frac{6(-1)^{i+1}}{(i+2) !}\left[(i+2) !((i+3) \alpha-n+2)+\prod_{l=j}^{i}(2 \alpha-n+j+1)\right) \\
& \times(i(2 \alpha-n+i+2)(2 \alpha-n+i+3)-(i+1)(i+2)(3 \alpha-n+2+i))]
\end{aligned}
$$


Remark 4.3.1. This last matrix shows the rank is always at least $n-3$, which means there is always at most a 1-dimensional non-trivial solution space.

\subsubsection{Cocycles of degree 5}

When $n=5$, we only get two formulae and thus our complete coefficient matrix is only a $2 \times 2$ matrix, so the rank is automatically less than or equal to $n-3=2$. Thus there exists a non-trivial solution, and we simply solve the matrix to find a solution then solve for the trivial cocycle and find a satisfactory cocycle not equivalent to the trivial cocycle, as done in the previous sections. This gives an equivalence class of:

$$
(\alpha-1) k^{5} \theta^{(0)}-k^{4} \theta^{(1)}-12 k^{3} \theta^{(2)}+24 k^{2} \theta^{(3)}, \text { for } \alpha-\beta=4 .
$$

\subsubsection{Cocycles of degree 6}

When $n=6$, the rank of the coefficient matrix will only be $(n-3)$ when $b_{1, n-3}^{\prime}=$ $b_{1, n-2}=0$. This means there is only a non-trivial solution where both polynomial coefficients 4.3.1), 4.3.2 are zero at the same time.

$$
\begin{gathered}
b_{1, n-2}=\frac{1}{3}\left(-n^{3}+7 \alpha n^{2}+3 n^{2}-16 \alpha^{2} n-9 \alpha n-2 n+12 \alpha^{3}+6 \alpha\right) \\
b_{1, n-3}^{\prime}=-n^{3}+6 \alpha n^{2}+3 n^{2}-12 \alpha^{2} n-6 \alpha n-2 n+8 \alpha^{3}+4 \alpha
\end{gathered}
$$

Solving for the roots of these polynomials, we get two shared roots of the form $\alpha=\frac{n \pm \sqrt{-2+3 n}}{2}$ which reduces to $\alpha=5$ and $\alpha=1$. Similar to the methods used in the previous section, there is a 1-dimensional non-trivial solution space 
given by the equivalence classes of:

$$
\begin{array}{r}
2 k^{5} \theta^{(1)}+10 k^{4} \theta^{(2)}+60 k^{3} \theta^{(3)}-120 k^{2} \theta^{(4)}, \text { for } \alpha=5, \beta=0 . \\
12 k^{6} \theta^{(0)}-22 k^{5} \theta^{(1)}+10 k^{4} \theta^{(2)}+60 k^{3} \theta^{(3)}-120 k^{2} \theta^{(4)}, \text { for } \alpha=1, \beta=-4 .
\end{array}
$$

\subsubsection{Cocycles of degree 7}

When $n=7$, the rank of the coefficient matrix will equal $(n-3)$ when $b_{1, n-3}^{\prime}=$ $b_{1, n-2}=b_{2, n-3}^{\prime}=b_{2, n-2}=0$. This means, there is only a non-trivial solution space when all four polynomial coefficients are zero at the same time.

$$
\begin{aligned}
b_{2, n-2}=-\frac{1}{6}( & 24 \alpha^{4}+60 \alpha^{3}-44 \alpha^{3} n+12 \alpha^{2}-98 \alpha^{2} n+30 \alpha^{2} n^{2} \\
& +30 \alpha-55 \alpha n+50 \alpha n^{2}-9 \alpha n^{3}-10 n+17 n^{2} \\
& \left.-8 n^{3}+n^{4}\right) \\
b_{2, n-3}^{\prime}=-\frac{1}{2}( & -10 n+20 \alpha+8 \alpha^{2}-38 \alpha n+17 n^{2}-72 \alpha^{2} n \\
+ & 42 \alpha n^{2}+40 \alpha^{3}-8 n^{3}-32 \alpha^{3} n-8 \alpha n^{3}+24 \alpha^{2} n^{2} \\
+ & \left.16 \alpha^{4}+n^{4}\right)
\end{aligned}
$$

Solving for the roots, 4.3.1), 4.3.2), 4.3.3), and 4.3.4 all share common roots of $\alpha=\frac{n \pm \sqrt{-2+3 n}}{2}$. This gives 2 cases of non-trivial cocycles

$$
\begin{aligned}
\text { For } \alpha=\frac{7+\sqrt{19}}{2}: & -\frac{22+\sqrt{19}}{4} k^{7} \theta^{(0)}+\frac{31+7 \sqrt{19}}{2} k^{6} \theta^{(1)} \\
& -(25+7 \sqrt{19}) k^{5} \theta^{(2)}+30 k^{4} \theta^{(3)}+120 k^{3} \theta^{(4)}-240 k^{2} \theta^{(5)} \\
\text { For } \alpha=\frac{7-\sqrt{19}}{2}: & -\frac{22-\sqrt{19}}{4} k^{7} \theta^{(0)}+\frac{31-7 \sqrt{19}}{2} k^{6} \theta^{(1)} \\
& -(25-7 \sqrt{19}) k^{5} \theta^{(2)}+30 k^{4} \theta^{(3)}+120 k^{3} \theta^{(4)}-240 k^{2} \theta^{(5)}
\end{aligned}
$$




\subsubsection{Cocycles of degree greater than or equal to 8}

When $n \geq 8$, the system will only admit non-trivial cocycles when

$b_{1, n-3}^{\prime}=b_{1, n-2}=b_{2, n-3}^{\prime}=b_{2, n-2}=b_{3, n-3}^{\prime}=b_{3, n-2}=\cdots=b_{n-5, n-3}^{\prime}=b_{n-5, n-2}=0$

If there exists a common solution to these formulae, then this solution must satisfy all of the first four formulae, which means that the solution must necessarily be of the form

$$
\alpha=\frac{n \pm \sqrt{-2+3 n}}{2}
$$

This is not a solution to the 5 th or 6th formulae 4.3.5, 4.3.6) as shown in 4.3.7) and (4.3.8). This means the rank of the matrix is always at least $(n-2)$ and thus this case never admits a non-trivial solution space.

$$
\begin{aligned}
b_{3, n-2}= & \frac{1}{60}\left(-172 n+516 \alpha+360 \alpha^{2}-1080 \alpha n+320 n^{2}\right. \\
& -2060 \alpha^{2} n+1115 \alpha n^{2}+1140 \alpha^{3}-185 n^{3}-1440 \alpha^{3} n \\
& -336 \alpha^{4} n+312 \alpha^{3} n^{2}-144 \alpha^{2} n^{3}+33 \alpha n^{4}-340 \alpha n^{3} \\
& \left.+1060 \alpha^{2} n^{2}+144 \alpha^{5}-3 n^{5}+720 \alpha^{4}+40 n^{4}\right) \\
b_{3, n-3}= & \frac{1}{20}\left(-172 n+344 \alpha+240 \alpha^{2}-760 \alpha n+320 n^{2}\right. \\
& -1500 \alpha^{2} n+930 \alpha n^{2}+760 \alpha^{3}-185 n^{3}-1040 \alpha^{3} n \\
& -240 \alpha^{4} n+240 \alpha^{3} n^{2}-120 \alpha^{2} n^{3}+30 \alpha n^{4}-300 \alpha n^{3} \\
& \left.+840 \alpha^{2} n^{2}+96 \alpha^{5}-3 n^{5}+480 \alpha^{4}+40 n^{4}\right)
\end{aligned}
$$


At $\alpha=\frac{n \pm \sqrt{-2+3 n}}{2}$,

$$
\begin{aligned}
b_{3, n-2}=\frac{1}{40}( & -2 n \mp 6 \sqrt{3 n-2} \pm 9 n \sqrt{3 n-2} \\
& \left.+3 n^{2} \mp 3 n^{2} \sqrt{3 n-2}-n^{3}\right) \\
b_{3, n-3}= \pm & \pm \frac{3 \sqrt{3 n-2}}{20}-(n-1) !
\end{aligned}
$$

\subsection{Dual Modules}

The dual vector space of a vector space $V$ is defined as

$$
V^{*}=\{\varphi: V \rightarrow \mathbb{C} ; \varphi \text { is linear }\}
$$

or $V^{*}$ is the set of all linear functionals on $V$.

If $(V, \rho)$ is a module for a Lie algebra $L$, then its dual vector space $V^{*}$ admits the action of $L$ in the following way:

$$
\left(\rho^{*}(x) \varphi\right) v=-\varphi(\rho(x) v)
$$

where $\rho^{*}$ is the action on the dual vector space.

When $V=T(\alpha, \gamma), T(\alpha, \gamma)^{*}$ contains the elements

$$
v_{m}^{*}: T(\alpha, \gamma) \rightarrow \mathbb{C}, v_{m}^{*}\left(v_{n}\right)=\delta_{m, n}
$$

For finite dimensional vector spaces, the dual vector space is isomorphic to the original space. In infinite dimensions though, the dual vector space is, in general, a much larger space. In this case, the dual vector space is larger than we want, 
so we introduce the restricted dual of $T(\alpha, \gamma)$, defined as $T(\alpha, \gamma)^{*}=\oplus_{m \in \gamma+\mathbb{Z}} \mathbb{C} v_{m}^{*}$. From now on, when we refer to the dual space, we mean the restricted dual space.

First, we would like to shift the basis of $T(\alpha, \gamma)^{*}$ to behave more like the original space. Let $\widetilde{v}_{m}=v_{-m}^{*}$. As $m \in \gamma+\mathbb{Z}$, then the basis $\widetilde{v}_{m}$ is indexed by $-\gamma+\mathbb{Z}$. The $W_{1}$-action on this basis is given by

$$
\begin{aligned}
\left(e_{k} \cdot \widetilde{v}_{m}\right) v_{m} & =-\widetilde{v}_{m}\left(e_{k} v_{s}\right) \\
& =-(s+\alpha k) v_{-m}^{*}\left(v_{s+k}\right) \\
& =-(s+\alpha k) \delta_{-m-s-k, 0} \\
& =-(-m-k+\alpha k) \delta_{-m-s-k, 0} \\
& =(m+(1-\alpha) k) \widetilde{v}_{m+k} v_{s} \\
\Longrightarrow e_{k} \widetilde{v}_{m} & =(m+(1-\alpha) k) \widetilde{v}_{m+k}
\end{aligned}
$$

As $W_{1}$-modules, $T(\alpha, \gamma)^{*} \cong T(1-\alpha,-\gamma)$ by the isomorphism $\widetilde{v}_{m}=v_{-m}^{*} \rightarrow v_{m}$. For the short exact sequence

$$
0 \rightarrow T(\alpha, \gamma) \rightarrow M \rightarrow T(\beta, \gamma) \rightarrow 0
$$

with the cocycle $\tau(k, m)$, we can compare the dual spaces of these modules. Then

$$
\begin{aligned}
e_{k} \widetilde{v}_{m} & =(m+(1-\alpha) k) \widetilde{v}_{m+k}-\tau(k,-m-k) \widetilde{w}_{m+k} \\
e_{k} \widetilde{w}_{m} & =(m+(1-\beta) k) \widetilde{w}_{m+k}
\end{aligned}
$$

As a consequence, we can relate the module extensions of the dual modules to module extensions of the original modules. The short exact sequence

$$
0 \rightarrow T(1-\beta,-\gamma) \rightarrow M^{*} \rightarrow T(1-\alpha,-\gamma) \rightarrow 0
$$


admits the cocycle $\tau^{*}(k, m)=-\tau(k,-m-k)$. Thus from finding one cocycle, we can easily derive another one by looking at the dual extension.

It is of note that for our equivalence classes of polynomial cocycles, the cases that give a range of possible values for $\alpha$ admit dual cocycles in the same equivalence class. The cases of degree 2, degree 6 and degree 7 cocycles do not admit a range of values for $\alpha$, but only have non-trivial cocycles for exactly two values of $\alpha$. In these cases, the dual cocycle is not in the same equivalence class as the original cocycle, but is of the same degree. Thus the two cases of degree 2 cocycles are dual to each other, the two cases of degree 6 cocycles are dual to each other and the two cases of degree 7 cocycles are also dual to each other. 


\section{Chapter 5}

\section{Delta Cocycles}

As discussed in Chapter 3, our cocycles may have a delta function of $\delta_{k+m, 0}$ when $\gamma \in \mathbb{Z}$ and $\alpha=0$. In this chapter, we will assume that $\alpha=0$ and $\gamma \in \mathbb{Z}$ and that our $\tau$-functions are delta functions. These cocycles will classify extensions of the form

$$
0 \rightarrow D(0,0) \rightarrow M \rightarrow T(\beta, 0) \rightarrow 0
$$

where $D(0,0)$ is the 1 -dimensional trivial submodule of $T(0,0)$ as defined in Chapter 2. As $D(0,0) \subset T(0,0)$, this extension will admit an extension

$$
0 \rightarrow T(0,0) \rightarrow M^{\prime} \rightarrow T(\beta, 0) \rightarrow 0
$$

In this section, we will find these delta cocycles in the context $M^{\prime}$ rather than $\widetilde{M}_{N}$, where $\delta_{k}\left(t^{m}\right)=\delta_{k+m, 0} v_{m+k}$.

These delta cocycles will be of the form

$$
\tau(k, m)=\delta_{m+k, 0} f(k, m)=\delta_{m+k, 0} f(k,-k)
$$

Thus, $f$ is a function of $k$. In fact, by Theorem 3.4 .1 for $e_{k} . w_{-k}$ and $\beta \neq 1, f(k)$ 
must be a polynomial function in $k$. As we will deal with the special case of $\beta=1$ in the next chapter, we will assume that $f(k)$ is a polynomial in $k$ for every $\beta$ for now.

The dual spaces also admit a delta function cocycle, given by the short exact sequence

$$
0 \rightarrow T(1-\beta, 0) \rightarrow M \rightarrow T(1,0) \rightarrow 0
$$

where $\tau(k, m)=\delta_{m, 0} g(k)$.

We will first handle the case that $\alpha=0$, then see what results we can obtain from the dual case.

\subsection{Conditions for delta functions}

As derived in Chapter 3, cocycles must satisfy the relation (3.1.2) so for $\tau(k, m)=$ $\delta_{k+m, 0} f(k)$,

$$
\begin{aligned}
(s-k) \delta_{k+m+s, 0} f(k+s)= & (m+s) \delta_{s+m, 0} f(s)-(m+k) \delta_{k+m, 0} f(k) \\
& +(m+\beta s) \delta_{k+m+s, 0} f(k)-(m+\beta k) \delta_{k+m+s, 0} f(s)
\end{aligned}
$$

This reduces to:

$$
(s-k) f(k+s)=((\beta-1) s-k) f(k)-((\beta-1) k-s) f(s)
$$

Recall a trivial cocycle is a 1-coboundary which becomes the zero function under some change of basis. Let $u_{m}=w_{m}+c \delta_{m, 0} v_{0}$ be a new basis for the module $M$. Then

$$
e_{k} u_{m}=(m+\beta k) w_{m}+c \delta_{m, 0} e_{k} v_{0}
$$




$$
\begin{aligned}
& =(m+\beta k) w_{m} \\
& =(m+\beta k) w_{m}+(m+\beta k) \delta_{m+k, 0} c v_{0}-(m+\beta k) \delta_{m+k, 0} c v_{0} \\
& =(m+\beta k) u_{m}+k(1-\beta) \delta_{m+k, 0} c v_{0}
\end{aligned}
$$

Thus $\tau(k, m)$ is trivial if there exists a constant $c \in \mathbb{C}$ such that

$$
\tau(k, m)=c k(1-\beta) \delta_{k+m, 0}
$$

To solve for cocycles $\tau(k, m)=\delta_{k+m, 0} f(k)$, we know that the function $f(k)$ must be polynomial so that for some $n \in \mathbb{N}, f(k)$ is given by

$$
f(k)=a_{n} k^{n}+a_{n-1} k^{n-1}+\cdots+a_{1} k+a_{0} .
$$

Equation (5.1.1) is homogeneous in $s$ and $k$ so each $f(k)=k^{m}$ can be treated separately.

When $n=0$, we only need to solve a simple linear equation for $a_{0}$ using (5.1.1):

$$
\begin{aligned}
(s-k) a_{0} & =((\beta-1) s-k) a_{0}-((\beta-1) k-s) a_{0} \\
& =(\beta s-s-k-\beta k+k+s) a_{0} \\
& =\beta(s-k) a_{0}
\end{aligned}
$$

So $f(k)=a_{0}$ is a valid function only when $\beta=1$. Since

$$
a_{0} \delta_{k+m, 0}=c k(1-\beta) \delta_{k+m, 0} \Longleftrightarrow a_{0}=0
$$

then $\delta_{k+m, 0}$ is non-trivial for $\beta=1$. This gives an equivalence class of $\delta_{k+m, 0}$ for $\beta=1$ 
When $n=1, f(k)=k$ so that

$$
\begin{aligned}
(s-k)(k+s) & =((\beta-1) s-k) k-((\beta-1) k-s) s \\
& =(s-k)(s+k)
\end{aligned}
$$

which holds for all $\beta$, but is only non-trivial when $\beta=1$. This gives an equivalence class of $\delta_{k+m, 0} k$ for $\beta=1$.

When $n=2, f(k)=k^{2}$ so that

$$
\begin{aligned}
(s-k)(k+s)^{2} & =((\beta-1) s-k) k^{2}-((\beta-1) k-s) s^{2} \\
& =(\beta)\left(s k^{2}-s^{2} k\right)+\left(s^{3}+s^{2} k-s k^{2}-k^{3}\right)
\end{aligned}
$$

which holds only if $\beta=0$. This gives an equivalence class of $\delta_{k+m, 0} k^{2}$ for $\beta=0$.

When $n=3, f(k)=k^{3}$ so that

$$
\begin{aligned}
(s-k)(k+s)^{3} & =((\beta-1) s-k) k^{3}-((\beta-1) k-s) s^{3} \\
& =(\beta+1)\left(s k^{3}-s^{3} k\right)+\left(s^{4}-2 s k^{3}+2 k s^{3}-k^{4}\right)
\end{aligned}
$$

which holds only if $\beta=-1$. This gives an equivalence class of $\delta_{k+m, 0} k^{3}$ for $\beta=-1$.

When $n \geq 4$, by 5.1 .1 we can obtain the system

$$
0=(s-k)(k+s)^{n}-((\beta-1) s-k) k^{n}+((\beta-1) k-s) s^{n}
$$

The coefficient at $s^{n-1} k^{2} n \geq 4$ will be given by:

$$
\left(\left(\begin{array}{l}
n \\
2
\end{array}\right)-\left(\begin{array}{l}
n \\
1
\end{array}\right)\right)=\frac{1}{2} n(n-1)-n \neq 0 \text { when } n \neq 3
$$

Thus, this coefficient is non-zero for any value of $\beta$. Therefore this system will only admit the trivial cocycle as a solution. 
We obtain the equivalence classes of:

$$
\begin{aligned}
\delta_{k+m, 0}, \text { when } \beta & =1 \\
\delta_{k+m, 0} k, \text { when } \beta & =1 \\
\delta_{k+m, 0} k^{2}, \text { when } \beta & =0 \\
\delta_{k+m, 0} k^{3}, \text { when } \beta & =-1
\end{aligned}
$$

This last cocycle $\delta_{k+m, 0} k^{3}$ for $\beta=-1$ corresponds to the Virasoro cocycle, the central extension of the Witt algebra described in Chapter 2. In this equivalence class is $\delta_{m+k} \frac{1}{12}\left(k^{3}-k\right)$, the more common form of the Virasoro cocycle.

Now, we can look at the dual extension.

$$
0 \rightarrow T(1-\beta, 0) \rightarrow M \rightarrow T(1,0) \rightarrow 0
$$

As was shown at the end of the previous section, $\tau^{*}(k, m)=\tau(k,-m-k)$ so that if $\tau(k, m)=\delta_{k+m, 0} f(k), \tau^{*}(k, m)=\delta_{k-m-k, 0} f(k)=\delta_{m, 0} f(k)$ when $\beta=1$. This gives us the following equivalence classes:

$$
\begin{array}{r}
\delta_{m, 0}, \text { when } \alpha=0, \beta=1 \\
\delta_{m, 0} k, \text { when } \alpha=0, \beta=1 \\
\delta_{m, 0} k^{2}, \text { when } \alpha=1, \beta=1 \\
\delta_{m, 0} k^{3}, \text { when } \alpha=2, \beta=1
\end{array}
$$

Notice here that when $\alpha=0, \beta=1$, we obtain both cocycles $\delta_{k+m, 0}$ and $\delta_{m, 0}$. As these two differ by the coboundary $\delta_{k+m, 0}-\delta_{m, 0}$ (4.1.6), these cocycles are contained in the same equivalence classes. Thus, (5.1.8) is not a new equivalence class. 


\section{Chapter 6}

\section{Special case of $\beta=1$}

The approach used in Chapter 3 to find conditions on our cocycles depended on the fact that $\beta \neq 1$. Recall that this method did not work because our homomorphism from $T(\beta, \gamma) \rightarrow \widehat{T(\beta, \gamma)}$ was not injective for $\beta=1$, so that there was no obvious

way in which to define $\widetilde{M}_{N}$. In particular, $\varepsilon_{j} \notin \widehat{T(1, \gamma)}$ for any $j \in \gamma+\mathbb{Z}$. We deal with this case here, using a similar approach by defining a basis of $M$ using the $A$-cover $\widehat{M}$.

From Chapter 4, we found all possible polynomial cocycles including the case of $\beta=1$. Now, we will assume they are strictly not polynomial and see what cocycles arise from this case.

\subsection{Conditions on non-polynomial cocycles}

We would like to find an appropriate basis of $M$ so that we can determine conditions on cocycles. First, introduce $\sigma_{0} \in \widehat{M}$ as $\sigma_{0}=\phi\left(e_{0}, w_{\gamma}\right)$. Then

$$
\sigma_{0}\left(t^{m}\right)=\phi\left(e_{0}, w_{\gamma}\right)\left(t^{m}\right)=e_{m} . w_{\gamma}=(m+\gamma) w_{m+\gamma}+\tau(m, \gamma) v_{m+\gamma}
$$


for all $m \in \mathbb{Z}, k \in \mathbb{Z}$.

Now, introduce a new basis of $M$ by

$$
\widehat{w}_{m+\gamma}=\frac{1}{m+\gamma} \sigma_{0}\left(t^{m}\right), \text { for } m+\gamma \neq 0
$$

Note that in the case of $\gamma \in \mathbb{Z}$, we have a basis for the module extension given by the short exact sequence

$$
0 \rightarrow T(\alpha, 0) \rightarrow M^{\prime} \rightarrow T^{\circ}(1,0) \rightarrow 0
$$

By Theorem 3.4.1, $\widehat{M} \cong A \otimes \widehat{M}_{\gamma}$ for some finite representation $\widehat{M}_{\gamma}$ of $\mathscr{L}_{+}$. Then $\sigma_{0}\left(t^{m}\right)=t^{m} \otimes \sigma$ for some $\sigma \in \widehat{M}_{\gamma}$. For $m+\gamma \neq 0$,

$$
\begin{aligned}
e_{k} . \widehat{w}_{m+\gamma} & =\frac{1}{m+\gamma} e_{k} \cdot\left(\sigma_{0}\left(t^{m}\right)\right) \\
& =\frac{1}{m+\gamma} e_{k} \cdot\left(t^{m} \otimes \sigma\right) \\
& =\frac{1}{m+\gamma}\left((m+\gamma) t^{m+k} \otimes \sigma+k u_{1}\left(t^{m+k}\right)+\cdots+k^{n} u_{n}\left(t^{m+k}\right)\right) \\
& =\frac{1}{m+\gamma}\left((m+\gamma) \sigma_{0}\left(t^{m+k}\right)+k u_{1}\left(t^{m+k}\right)+\cdots+k^{n} u_{n}\left(t^{m+k}\right)\right) \\
& =(m+k+\gamma) \widehat{w}_{m+k+\gamma}+\frac{1}{m+\gamma}\left(k u_{1}\left(t^{m+k}\right)+\cdots+k^{n} u_{n}\left(t^{m+k}\right)\right)
\end{aligned}
$$

for all $k \in \mathbb{Z}$, where $u_{i}=\frac{1}{i !} \rho\left(z^{i} \frac{d}{d z}\right) \sigma$ as in Theorem 3.4.1. Note that for $\gamma \in \mathbb{Z}, \sigma_{0}(1)=\tau(0,0) v_{0} \in \widehat{M}(\alpha, 0)$. Then the image of $\sigma_{0}$ under the map $\widehat{\pi}: \widehat{M} \rightarrow \widehat{T(1, \gamma)}$ is simply zero since

$$
\begin{aligned}
\left(e_{k} \widehat{\pi}\left(\sigma_{0}\right)\right)\left(t^{m}\right) & =\left(e_{k} \psi\left(e_{0}, \bar{w}_{\gamma}\right)\right)\left(t^{m}\right) \\
& =e_{k}\left(\psi\left(e_{0}, \bar{w}_{\gamma}\right) t^{m}\right)-\psi\left(e_{0}, \bar{w}_{\gamma}\right)\left(e_{k} t^{m}\right) \\
& =(m+\gamma)(m+\gamma+k) w_{m+\gamma+k}-(m+\gamma)(m+\gamma+k) w_{m+\gamma+k} \\
& =0, \forall m \in \gamma+\mathbb{Z}
\end{aligned}
$$


so that

$$
e_{k} \phi\left(e_{0}, \bar{w}_{\gamma}\right)=0 \Longrightarrow \phi\left(e_{0}, \bar{w}_{\gamma}\right)=0
$$

and so $\widehat{\pi}\left(\sigma_{0}\right) \in \widehat{M}(\alpha, \gamma)$. This shows that $k u_{1}+\cdots+k^{n} u_{n} \in \widehat{M}(\alpha, \gamma)$, so that for $m \in \gamma+\mathbb{Z}$, the cocycle $\widetilde{\tau}(k, m)=\frac{1}{m}\left(k u_{1}+\cdots+k^{n} u_{n}\right), m \widetilde{\tau}(k, m)$ is contained in a submodule of the form described in Proposition 3.3.2. In other words, this cocycle will be a polynomial function or a $\delta_{m+k, 0}$ function when $\alpha=0$, with a factor of $m^{-1}$. Notice here that we may apply Theorem 3.4.1 to obtain that if a cocycle is given by $\frac{1}{m} \delta_{m+k, 0} f(k), f(k)$ is polynomial in $k$.

The function $\widehat{\pi}(\widetilde{\tau})=\tau^{\prime}(k, m)$ is not defined when $m=0$. This $\tau$-function completely determines the cocycle on the short exact sequence with $T^{\circ}(1,0)$. To extend this cocycle onto $M$, we introduce a possible $\delta_{m, 0}$ function to determine the cocycle on the basis vector $w_{0}$.

$$
e_{k} w_{0}=k \widehat{w_{k}}+\mu(k, 0) v_{k}
$$

Note that $\mu(k, 0)$ is simply a function on $k$, and so we will use the notation $\mu(k)$.

We can extend the cocycle to the whole space of $M$ by setting $\tau(k, m)$ to be the piecewise function:

$$
\tau(k, m)= \begin{cases}\tau^{\prime}(k, m), & m \neq 0 \\ \mu(k), & m=0\end{cases}
$$

These two components are independent so that it admits two cases, when the cocycle is of the form $\tau^{\prime}(k, m)$ or when $\tau(k, m)=\delta_{m, 0} \mu(k)$. Hence, for $\beta=1$, cocycles in $M$ can be polynomial functions in $k$ with a possible factor of $m^{-1}$, $\delta_{k+m, 0}$ functions with a possible factor of $m^{-1}$ or $\delta_{m, 0}$ functions. 


\subsection{Cocycles with a factor of $m^{-1}$}

Suppose that $\tau^{\prime}(k, m)=m^{-1} \mu(k, m)$ for $k \in \mathbb{Z}, m \in \gamma+\mathbb{Z}$. If this is a cocycle then by 3.1 .2 ,

$$
\begin{aligned}
(s-k) m^{-1} \mu(k+s, m)= & (m+s+\alpha k) m^{-1} \mu(s, m)+(m+s)(m+s)^{-1} \mu(k, m+s) \\
& -(m+k+\alpha s) m^{-1} \mu(k, m)-(m+k)(m+k)^{-1} \mu(s, m+k) \\
(s-k) \mu(k+s, m)= & (m+s+\alpha k) \mu(s, m)+m \mu(k, m+s) \\
& -(m+k+\alpha s) \mu(k, m)-m \mu(s, m+k)
\end{aligned}
$$

So this reduces to the case that $\mu(k, m)$ is a cocycle for $\beta=0$.

In the case that $\alpha=0$ and $\mu(k, m)=\delta_{k+m, 0} f(k)$ there are two cocycles: the trivial cocycle $\delta_{k+m, 0} k$ and the nontrivial cocycle $\delta_{k+m, 0} k^{2}$.

$$
\delta_{k+m, 0} m^{-1} k=\delta_{m+k} \frac{k}{-k}=-\delta_{k+m, 0}
$$

This first case gives a cocycle of the form $\delta_{k+m, 0}$ which was found to be non-trivial in Chapter 5.

$$
\delta_{k+m, 0} m^{-1} k^{2}=\delta_{k+m, 0} \frac{k^{2}}{-k}=-k \delta_{k+m, 0}
$$

The second case is exactly the equivalence class of $\delta_{k+m, 0} k$ found in Chapter 5 .

In the case that $\mu(k, m)$ is a polynomial function, then we would like to see when this cocycle is a coboundary. Then there exits some change of basis of $M$ such that the cocycle becomes trivial. In other words, there exists $g: \gamma+\mathbb{Z} \rightarrow \mathbb{C}$ such that

$$
g(m)(m+\alpha k)-g(m+k)(m+k)=\frac{1}{m} \mu(k, m)
$$


But if $k g(k)=f(k)$, then this reduces to

$$
\begin{array}{r}
m g(m)(m+\alpha k)-m g(m+k)(m+k)=\mu(k, m) \\
f(m)(m+\alpha k)-f(m+k) m=\mu(k, m)
\end{array}
$$

which is the same condition for $\mu(k, m)$ being a non-trivial polynomial cocycle for $\beta=0$. In Chapter 4 , we described all polynomial cocycles in $\widetilde{M}_{N}$. In Chapter 7 , we describe the resulting polynomial cocycles in $M$. Then using Table $7.2, \mu(k, m)$ can be any of the following:

$$
\begin{array}{lc}
\alpha=0, & k \\
\alpha=1, & k m \\
\alpha=1, & k^{2} \\
\alpha=2, & k^{3}+k^{2} m \\
\alpha=3, & k^{3} m+k^{2} m^{2} \\
\alpha=4, & k^{4} m-6 k^{3} m^{2}-4 k^{2} m^{3} \\
\alpha=5, & 2 k^{5} m-5 k^{4} m^{2}+10 k^{3} m^{3}+5 k^{2} m^{4}
\end{array}
$$

Most of these cases will reduce to equivalence classes from Chapter 4. Four cases admit new partial polynomial cocycles, giving the equivalence classes

$$
\begin{aligned}
& \alpha=0, \beta=1, \quad m^{-1} k \\
& \alpha=1, \beta=1, \quad m^{-1} k^{2} \\
& \alpha=2, \beta=1, \quad m^{-1} k^{3}+k^{2}
\end{aligned}
$$

Remark 6.2.1. These functions are not polynomial but can be considered such under some change of basis.

Consider the homomorphism from $T(0, \gamma) \rightarrow T(1, \gamma)$ by $v_{m} \rightarrow m w_{m}$. For 
$\gamma \notin \mathbb{Z}$, this map is bijective so that $T(0, \gamma) \cong T(1, \gamma)$. In the case that $\gamma \in \mathbb{Z}$, the image of this map is $T^{\circ}(1,0)$ and the kernel is $\mathbb{C} v_{0}=D(0,0)$ so that

$$
T(0,0) / D(0,0) \cong T^{\circ}(1,0)
$$

But as these $\tau^{\prime}$-functions zero at $m=0$, they can be considered cocycles defined on $T^{\circ}(1,0)$.

By applying this map to $w_{m} \in T(1, \gamma)$ to $w_{m}^{\prime} \in T(0, \gamma)$,

$$
\begin{aligned}
e_{k} \cdot w_{m} & =(k+m) w_{m+k}+\frac{1}{m} \mu(k, m) v_{m+k} \\
m e_{k} \cdot w_{m} & =m(k+m) w_{m+k}+\mu(k, m) v_{m+k} \\
e_{k} \cdot w_{m}^{\prime} & =m w_{m}^{\prime}+\mu(k, m) v_{m+k}
\end{aligned}
$$

and $\mu(k, m)$ is a polynomial cocycle. Although these module extensions are not identical, they are equivalent under some change of basis so that these cocycles can be thought of as polynomials in some sense.

\subsection{Delta cocycles}

As in Chapter 5, we will consider delta functions of the form $\delta_{m, 0} f(k, m)$. This reduces to $f(k, m)=\mu(k)$ where $\mu$ is polynomial in $k$. If we take the change of basis $w_{0}^{\prime}=w_{0}+v_{0}$, then we find that $\delta_{m, 0} \alpha k$ is the trivial cocycle.

Now, we take $\tau(k, m)=\delta_{m, 0} \mu(k)$ so that the $W_{1}$-action on $M$ is given by

$$
\begin{aligned}
e_{k} w_{0} & =k w_{k}+\mu(k) v_{k} \\
e_{k} w_{m} & =(m+k) w_{m+k}
\end{aligned}
$$


By the cocycle condition 3.1 .2 ,

$$
(k-s) \mu(k+s)+(k+\alpha s) \mu(k)-(s+\alpha k) \mu(s)=0
$$

Using this condition, we may derive the following equations:

For $k=0, s=1$ :

$\alpha \mu(0)=0$

For $k=1, s=2$ :

$$
\mu(3)=(2+\alpha) \mu(2)-(1+2 \alpha) \mu(1)
$$

For $k=1, s=3$ :

$$
\mu(4)=\frac{1}{2}(3+\alpha)(2+\alpha) \mu(2)-\left(2+5 \alpha+\alpha^{2}\right) \mu(1)
$$

For $k=1, s=4: \quad \mu(5)=\frac{1}{6}(4+\alpha)(3+\alpha)(2+\alpha) \mu(2)-\frac{1}{3}\left(9+26 \alpha+9 \alpha^{2}+\alpha^{3}\right) \mu(1)$

For $k=2, s=3$ :

$$
\mu(5)=\left(4+4 \alpha+2 \alpha^{2}\right) \mu(2)-\left(3+8 \alpha+4 \alpha^{2}\right) \mu(1)
$$

By equating the last two equations, we obtain the result that

$$
\left(2 \alpha-3 \alpha^{2}+\alpha^{3}\right) \mu(2)=2\left(2 \alpha-3 \alpha^{2}+\alpha^{3}\right) \mu(1)
$$

As $2 \alpha-3 \alpha^{2}+\alpha^{3}=\alpha(\alpha-1)(\alpha-2)$, then $\mu(2)=2 \mu(1)$ as long as $\alpha \neq 0,1,2$.

Lemma 6.3.1. If $\alpha \neq 0,1,2$, then $\mu(n)=n \mu(1)$ for $n \geq 3$.

Proof. If $n=3$, then $\mu(3)=(2(2+\alpha)-(1+2 \alpha)) \mu(1)=3 \mu(1)$.

By induction, set $s=n, k=1$, then

$$
\begin{aligned}
\mu(n+1) & =\frac{1}{n-1}((n+\alpha) \mu(n)-(1+n \alpha) \mu(1)) \\
& =\frac{1}{n-1}((n+\alpha) n-(1+n \alpha)) \mu(1) \\
& =\frac{1}{n-1}\left(n^{2}-1\right) \mu(1) \\
& =(n+1) \mu(1)
\end{aligned}
$$


Thus by the above recurrence relation $\mu(k)=k$ except when $\alpha=0,1$ or 2 . In the next three lemmas, we consider $\mu(k)$ in these three special cases.

Lemma 6.3.2. If $\alpha=0$, then $\mu(n)=(n-1) \mu(2)-(n-2) \mu(1)$ for $n \geq 3$.

Proof. If $n=3$, then $\mu(3)=2 \mu(2)-\mu(1)$.

By induction, set $s=n, k=1$, then

$$
\begin{aligned}
\mu(n+1) & =\frac{1}{n-1}((n) \mu(n)-\mu(1)) \\
& =\frac{1}{n-1}((n(n-1)) \mu(2)-(n(n-2)+1) \mu(1)) \\
& =n \mu(2)-\frac{1}{n-1}\left(n^{2}-2 n+1\right) \mu(1) \\
& =n \mu(2)-(n-1) \mu(1)
\end{aligned}
$$

In this case, $\mu(k)$ will be a polynomial of degree 1 .

Lemma 6.3.3. If $\alpha=1$, then $\mu(n)=\frac{n(n-1)}{2} \mu(2)-\left(n^{2}-2 n\right) \mu(1)$ for $n \geq 3$.

Proof. If $n=3$, then $\mu(3)=3 \mu(2)-3 \mu(1)$, so that

$$
\frac{n(n-1)}{2} \mu(2)-\left(n^{2}-2 n\right) \mu(1)=3 \mu(2)-3 \mu(1)=\mu(3)
$$

By induction, set $s=n, k=1$, then

$$
\begin{aligned}
& \mu(n+1)=\frac{1}{n-1}((n+1) \mu(n)-(n+1) \mu(1)) \\
& \mu(n+1)=\frac{1}{n-1}\left(\left((n+1) \frac{n(n-1)}{2}\right) \mu(2)-\left((n+1)\left(n^{2}-2 n\right)+(n+1)\right) \mu(1)\right) \\
& \mu(n+1)=\frac{n(n+1)}{2} \mu(2)-\frac{1}{n-1}\left(n^{3}-n^{2}-n+1\right) \mu(1) \\
& \mu(n+1)=\frac{n(n+1)}{2} \mu(2)-\left(n^{2}-1\right) \mu(1)
\end{aligned}
$$




$$
\mu(n+1)=\frac{n(n+1)}{2} \mu(2)-\left((n+1)^{2}-2(n+1)\right) \mu(1)
$$

Hence, $\mu(k)$ is a polynomial of degree 2 .

Lemma 6.3.4. If $\alpha=2$, then $\mu(n)=\frac{n^{3}-n}{6} \mu(2)-\frac{n^{3}-4 n}{3} \mu(1)$ for $n \geq 3$.

Proof. If $n=3$, then $\mu(3)=4 \mu(2)-5 \mu(3)$, so that

$$
\frac{n^{3}-n}{6} \mu(2)-\frac{n^{3}-4 n}{3} \mu(1)=4 \mu(2)-5 \mu(1)=\mu(3)
$$

By induction, set $s=n, k=1$, then

$$
\begin{aligned}
\mu(n+1)= & \frac{1}{n-1}\left((n+2) \frac{n(n+1)(n-1)}{6} \mu(2)\right. \\
& \left.-\left(\frac{(n+2)\left(n^{3}-4 n\right)}{3}+(2 n+1)\right) \mu(1)\right) \\
\mu(n+1)= & \left.\frac{n(n+1)(n+2)}{6} \mu(2)-\frac{1}{3}\left(n^{4}+2 n^{3}-4 n^{2}-8 n+6 n+3\right)\right) \mu(1) \\
\mu(n+1)= & \frac{(n+1)^{2}-(n+1)}{6} \mu(2)-\frac{1}{3}\left(n^{4}+2 n^{3}-4 n^{2}-2+3\right) \mu(1) \\
\mu(n+1)= & \frac{(n+1)^{2}-(n+1)}{6} \mu(2)-\frac{(n+1)^{3}-4(n+1)}{3} \mu(1)
\end{aligned}
$$

This last case gives the result that $\mu(k)$ is a polynomial of degree 3 .

Thus if $\mu(k)$ is non-trivial, then it has possible values of:

$$
\begin{aligned}
& \alpha=0, \quad \mu(k)=k \text { or } 1 \\
& \alpha=1, \quad \mu(k)=k^{2} \\
& \alpha=2, \quad \mu(k)=k^{3}
\end{aligned}
$$

which are exactly the dual delta function cocycles we found in Chapter 5. 


\section{Chapter 7}

\section{Conclusion}

In Chapter 4, we found all possible $\widetilde{\tau}(k, m)$ cocycles contained in the span of $\left\{\theta_{k}^{(0)}, \ldots, \theta_{k}^{(N)}\right\}$, which are summarized below. These functions yield polynomial cocycles in $M$ under the map $\pi: \widetilde{M}_{N} \rightarrow M$, which are listed in Table 7.2. Recall that these cocycles determine the action of the Witt algebra on the module $M$, which precisely determines the modules $M$ so that these results give us a classification of all length two extensions of tensor modules.

Most extensions admit classes of cocycles for a range of $\alpha$ and $\beta$. The few exceptions occur in pairs; these pairs are modules extensions that are dual to each other in the sense that for the extension $M / T(\alpha, \gamma) \cong T(\beta, \gamma)$, the dual extension is given by $M^{\prime} / T(1-\beta,-\gamma) \cong T(1-\alpha,-\gamma)$ with the dual cocycle $\tau^{*}(k, m)=\tau(k,-m-k)$. In the case where a range of $\alpha$ and $\beta$ are possible, these extensions are dual to themselves.

These polynomial functions have at most degree 7 . This comes from the equations we found that determine the coefficients $c_{i}$ in Lemma 4.1.3. When $n$ gets large, we quickly obtain systems that overdetermine the coefficients. The point when $n \geq 8$ is precisely where there is no non-trivial solution to the whole system 
of equations.

Table 7.1: Polynomial cocycles in $\widetilde{M}_{N}$

\begin{tabular}{|l|l|c|}
\hline$\alpha-\beta=0$ & $n=1$ & $k \theta^{(0)}$ \\
\hline$\alpha=1, \beta=0$ & $n=2$ & $k \theta^{(1)}$ \\
\hline$\alpha=1, \beta=0$ & $n=2$ & $k^{2} \theta^{(0)}$ \\
\hline$\alpha-\beta=2$ & $n=3$ & $k^{3} \theta^{(0)}-2 k^{2} \theta^{(1)}$ \\
\hline$\alpha-\beta=3$ & $n=4$ & $k^{3} \theta^{(1)}-2 k^{2} \theta^{(2)}$ \\
\hline$\alpha-\beta=4$ & $n=5$ & $(\alpha-1) k^{5} \theta^{(0)}-k^{4} \theta^{(1)}-12 k^{3} \theta^{(2)}+24 k^{2} \theta^{(3)}$ \\
\hline$\alpha=1, \beta=-4$ & $n=6$ & $2 k^{5} \theta^{(1)}+10 k^{4} \theta^{(2)}+60 k^{3} \theta^{(3)}-120 k^{2} \theta^{(4)}$ \\
\hline$\alpha=5, \beta=0$ & $n=6$ & $12 k^{6} \theta^{(0)}-22 k^{5} \theta^{(1)}+10 k^{4} \theta^{(2)}+60 k^{3} \theta^{(3)}-120 k^{2} \theta^{(4)}$ \\
\hline$\alpha=\frac{7+\sqrt{19}}{2}$, & $n=7$ & $-\frac{22+\sqrt{19}}{4} k^{7} \theta^{(0)}+\frac{31+7 \sqrt{19}}{2} k^{6} \theta^{(1)}-(25+7 \sqrt{19}) k^{5} \theta^{(2)}$ \\
$\beta=-\frac{5+\sqrt{19}}{2}$ & & $+30 k^{4} \theta^{(3)}+120 k^{3} \theta^{(4)}-240 k^{2} \theta^{(5)}$ \\
\hline$\alpha=\frac{7-\sqrt{19}}{2}$, & $n=7$ & $-\frac{22-\sqrt{19}}{4} k^{7} \theta^{(0)}+\frac{31-7 \sqrt{19}}{2} k^{6} \theta^{(1)}-(25-7 \sqrt{19}) k^{5} \theta^{(2)}$ \\
$\beta=-\frac{5-\sqrt{19}}{2}$ & & $+30 k^{4} \theta^{(3)}+120 k^{3} \theta^{(4)}-240 k^{2} \theta^{(5)}$ \\
\hline
\end{tabular}

There are a few remarks we can make about these functions in Table 7.2. By directly applying the map $\pi: \widetilde{M}_{N} \rightarrow M$, we do not obtain exactly the same cocycle, only a cocycle that is in the same equivalence class. This will not change the equivalence class though, so that we can shift the representative of the class to a function of the same form. The choice of these equivalence classes are a bit arbitrary; they are modelled after the results of Feigin and Fuks [8], There is a typo in [8] in the case of the degree 7 cocycles, otherwise we obtain the same results in Table 7.2 .

At the beginning of Chapter 4, we stated that it was enough to look at homogeneous polynomial cocycles. To obtain a general cocycle, we may have nonhomogeneous polynomials where each homogeneous component will be in one of these equivalence classes. The values of $\alpha$ and $\beta$ therefore put some limitations on the general cocycles. For example, there will not exist a cocycle that has both 
a non-trivial degree 6 component and a non-trivial degree 7 component.

Table 7.2: Polynomial cocycles in $M$

\begin{tabular}{|l|l|c|}
\hline$\alpha-\beta=0$ & $n=1$ & $k$ \\
\hline$\alpha=1, \beta=0$ & $n=2$ & $k m$ \\
\hline$\alpha=1, \beta=0$ & $n=2$ & $k^{2}$ \\
\hline$\alpha-\beta=2$ & $n=3$ & $k^{3}+2 k^{2} m$ \\
\hline$\alpha-\beta=3$ & $n=4$ & $k^{3} m+k^{2} m^{2}$ \\
\hline$\alpha-\beta=4$ & $n=5$ & $(\alpha-4) k^{5}+k^{4} m-6 k^{3} m^{2}-4 k^{2} m^{3}$ \\
\hline$\alpha=1, \beta=-4$ & $n=6$ & $12 k^{6}+22 k^{5} m+5 k^{4} m^{2}-10 k^{3} m^{3}-5 k^{2} m^{4}$ \\
\hline$\alpha=5, \beta=0$ & $n=6$ & $2 k^{5} m-5 k^{4} m^{2}+10 k^{3} m^{3}+5 k^{2} m^{4}$ \\
\hline$\alpha=\frac{7+\sqrt{19}}{2}$, & $n=7$ & $-\frac{22+\sqrt{19}}{4} k^{7}-\frac{31+7 \sqrt{19}}{2} k^{6} m$ \\
$\beta=-\frac{5+\sqrt{19}}{2}$ & & $-\frac{25+7 \sqrt{19}}{2} k^{5} m^{2}-5 k^{4} m^{3}+5 k^{3} m^{4}+2 k^{2} m^{5}$ \\
\hline$\alpha=\frac{7-\sqrt{19}}{2}$, & $n=7$ & $-\frac{22-\sqrt{19}}{4} k^{7}-\frac{31-7 \sqrt{19}}{2} k^{6} m$ \\
$\beta=-\frac{5-\sqrt{19}}{2}$ & & $-\frac{25-7 \sqrt{19}}{2} k^{5} m^{2}-5 k^{4} m^{3}+5 k^{3} m^{4}+2 k^{2} m^{5}$ \\
\hline
\end{tabular}

From the cocycle condition in given by $(3.1 .2)$, other extensions can be found. For example, the function $\tau(k, m)=1$ will satisfy the cocycle condition when $\alpha=\beta$. The action of $e_{0}$ on the basis vectors will be

$$
\begin{array}{r}
e_{0} v_{m}=m v_{m} \\
e_{0} w_{m}=m w_{m}+v_{m}
\end{array}
$$

This cocycle does not produce a weight module as $e_{0}$ will not act diagonally. Equation (3.1.2) is not enough to obtain a weight extension, while the $\widetilde{\tau}$ cocycles are in one-to-one correspondence to $\tau$ cocycles that yield weight module extensions.

For $\beta=1, \alpha=0$ and $\gamma \in \mathbb{Z}$, we obtained a few non-polynomial cocycles. Recall that for $\tau(k, m)=\frac{1}{m} \mu(k, m)$, where $\tau(k, 0)$ is defined to be zero. As was discussed in Section 6.2, these functions are almost polynomial in the sense that 
they admit polynomial cocycles under the change of basis $T(0, \gamma) \rightarrow T(1, \gamma)$.

Table 7.3: Non-polynomial cocycles

\begin{tabular}{|c|c|c|}
\hline$\gamma \in \mathbb{Z}$ & $\alpha=0, \beta=-1$ & $\delta_{k+m, 0} k^{3}$ \\
\hline$\gamma \in \mathbb{Z}$ & $\alpha=0, \beta=0$ & $\delta_{k+m, 0} k^{2}$ \\
\hline$\gamma \in \mathbb{C}$ & $\alpha=0, \beta=1$ & $m^{-1} k$ \\
\hline$\gamma \in \mathbb{Z}$ & $\alpha=0, \beta=1$ & $\delta_{k+m, 0}$ \\
\hline$\gamma \in \mathbb{Z}$ & $\alpha=0, \beta=1$ & $\delta_{k+m, 0} k$ \\
\hline$\gamma \in \mathbb{Z}$ & $\alpha=0, \beta=1$ & $\delta_{m, 0} k$ \\
\hline$\gamma \in \mathbb{C}$ & $\alpha=1, \beta=1$ & $m^{-1} k^{2}$ \\
\hline$\gamma \in \mathbb{Z}$ & $\alpha=1, \beta=1$ & $\delta_{m, 0} k^{2}$ \\
\hline$\gamma \in \mathbb{C}$ & $\alpha=2, \beta=1$ & $m^{-1} k^{3}+k^{2}$ \\
\hline$\gamma \in \mathbb{Z}$ & $\alpha=2, \beta=1$ & $\delta_{m, 0} k^{3}$ \\
\hline
\end{tabular}

These delta cocycles are of interest for another reason. Using the delta functions that give an extra factor on $v_{0}$ in the $W_{1}$-action on $T(\beta, \gamma)$, i.e. cocycles of the form $\delta_{k+m, 0} \mu(k)$, we can construct new Lie algebras.

Let $L$ be the Virasoro algebra which is spanned by $\left\{L(m), c_{1}\right\}$ where $c_{1}$ is the central extension, $m \in \mathbb{N}$. Suppose that module $V$ is a module for the Virasoro algebra with basis $\left\{W(m), c_{3}\right\}$. By viewing $V$ as a (possibly abelian) Lie algebra, we can look at the semi-direct product of $L$ with $V$ spanned by $\left\{L(m), W(m), c_{1}, c_{2}, c_{3}\right\}$ with bracket:

$$
\begin{aligned}
{[L(k), L(m)] } & =(m-k) L(k+m)+\delta_{k+m, 0} \frac{k^{3}-k}{12} c_{1} \\
{[L(k), W(m)] } & =(m-\beta k) W(k+m)+\delta_{k+m, 0} \mu(k) c_{2}
\end{aligned}
$$

where $V$ is a subalgebra and the bracket of any element with a central extension $c_{1}, c_{2}, c_{3}$ is simply zero.

In this way, we can construct what is called the $W(2,2)$ algebra [17]. Take $V$ 
to be the module corresponding to the adjoint representation of $L$ and let $V$ be an abelian Lie algebra. Then the bracket of the new algebra is given by

$$
\begin{aligned}
{[L(k), L(m)] } & =(m-k) L(m+k)+\delta_{k+m, 0} \frac{k^{3}-k}{12} c_{1} \\
{[L(k), W(m)] } & =(m-k) W(m+k)+\delta_{k+m, 0} \frac{k^{3}-k}{12} c_{1} \\
{[W(k), W(m)] } & =0
\end{aligned}
$$

This corresponds to the cocycle $\delta_{k+m, 0} k^{3}, \beta=-1$.

Similarly, we can construct the twisted Heisenberg-Virasoro algebra [4], Here, $V$ is the Heisenberg Lie algebra which is itself an $L$-module.

$$
\begin{aligned}
{[L(k), L(m)] } & =(m-k) L(m+n)+\delta_{k+m, 0} \frac{k^{3}-k}{12} c_{1} \\
{[L(k), W(m)] } & =m W(k+m)+\delta_{k+m, 0}\left(k^{2}-k\right) c_{2} \\
{[W(k), W(m)] } & =\delta_{k+m, 0} n c_{3}
\end{aligned}
$$

This corresponds to the cocycle $\delta_{k+m, 0} k^{2}, \beta=0$.

We can construct two more algebras of this form, given by the cases of $\delta_{k+m, 0}, \beta=$ $1, \delta_{k+m, 0} k, \beta=1$. As in the previous cases, $V$ can be taken as an abelian algebra or as a Heisenberg algebra. In the case that $V$ is a Heisenberg algebra, this construction is only a Lie algebra if $\beta=0$.

For the cocycle $\delta_{k+m, 0}, \beta=1$, we take $V$ to be an abelian Lie algebra. The resulting algebra is given below, where the bracket with any central element is trivial.

$$
\begin{aligned}
{[L(k), L(m)] } & =(m-k) L(m+n)+\delta_{k+m, 0} \frac{k^{3}-k}{12} c_{1} \\
{[L(k), W(m)] } & =(m+k) W(k+m)+\delta_{m+k, 0} c_{2} \\
{[W(k), W(m)] } & =0
\end{aligned}
$$


The case of $\delta_{k+m, 0} k, \beta=1$ will construct the algebra given by the following, where the the bracket with any central element is trivial.

$$
\begin{aligned}
{[L(k), L(m)] } & =(m-k) L(m+n)+\delta_{k+m, 0} \frac{k^{3}-k}{12} c_{1} \\
{[L(k), W(m)] } & =(m+k) W(k+m)+\delta_{m+k, 0} k c_{2} \\
{[W(k), W(m)] } & =0
\end{aligned}
$$

Notice that if we set $I(m)=m W(m)$,

$$
[L(k), I(m)]=m I(m+k)-m^{2} \delta_{m+k, 0}
$$

This action is very close to the twisted Heisenberg-Virasoro algebra. The difference is the action on $W(0)$ is given by $[L(k), W(0)]=k W(k)$.

The corresponding Virasoro modules are given by the extensions

$$
\begin{aligned}
& V / D(0,0) \cong T(0,0), \text { for } \delta_{k+m, 0} \\
& V / D(0,0) \cong T(1,0), \text { for } \delta_{k+m, 0} k
\end{aligned}
$$

The main goal of this work was to produce an explicit classification of length two module extensions of the Witt algebra. However, this method is promising in finding a similar classification of length two module extensions of the solenoidal subalgebra (see Definition 2.2 of [6]] of $W_{n}$, and may even be used to find a classification of module extensions of this type for $W_{n}$. 


\section{References}

[1] E. Arbarello, C. De Concini, V.G. Kac, C. Procesi, Moduli spaces of curves and representation theory, Comm. Math. Phys., 117, 1-36 (1988).

[2] A.A. Belavin, A. M. Polyakov, A.B. Zamolodchikov, Infinite conformal symmetry in two-dimensional quantum field theory, Nuclear Physics B, 241, No. $2,333-380(1984)$

[3] Y. Billig, Jet Modules, Canad. J. Math, 59, No.4, 712-729 (2007).

[4] Y. Billig, Representations of the twisted Heisenberg-Virasoro algebra at level zero, Canadian Mathematical Bulletin, 46, 529-537 (2003).

[5] Y. Billig, V. Futorny, Classification of simple $W_{n}$-modules with finite-dimensional weight spaces, arXiv:1304.5458 [math.RT]

[6] Y. Billig, V. Futorny, Classification of simple cuspidal modules for solenoidal Lie algebras, arXiv:1306.5478 [math.RT]

[7] C. H. Conley, Bounded Length 3 Representations of the Virasoro Lie Algebra, Internat. Math. Res. Notices, No. 12, 609-628 (2001).

[8] B. L. Feigin, D. B. Fuks, Homology of the Lie algebra of vector fields on the line, Funct. Anal. Appl., 14, No.3, 201-212 (1980). 
[9] D.B. Fuks, Cohomology of Infinite-dimensional Lie Algebras, Consultants Bureau, New York, 1986.

[10] J. Fuchs, Affine Lie algebras and quantum groups, Cambridge University Press, New York, 1992.

[11] James E. Humphreys, Introduction to Lie algebras and Representation Theory, Springer-Verlag, New York, 1972.

[12] V. G. Kac, A. K. Raina, N. Rozhkovskaya, Bombay lectures of highest weight representations of infinite dimensional Lie algebras, Second Edition, World Scientific Publishing Co. Pte. Ltd, Singapore, 2013.

[13] C. Martin, A. Piard, Indecomposable modules over the Virasoro Lie algebra and a conjecture of V. Kac, Commun. Math. Phys., 137, 109-132 (1991).

[14] C. Martin, A. Piard, Classification of the indecomposable bounded admissible modules over the Virasoro Lie algebra with weightspaces of dimension not exceeding two, Commun. Math. Phys., 150, 465-493 (1992).

[15] O. Mathieu, Classification of Harish-Chandra modules over the Virasoro Lie algebra, Invent. math., 107, 225-234 (1992).

[16] R. Moody, A. Pianzola, Lie Algebras with Triangular Decompositions, Wiley, New York, 1995.

[17] W. Zhang, C. Dong, $W$-algebra $W(2,2)$ and the vertex operator algebra $L\left(\frac{1}{2}, 0\right) \otimes L\left(\frac{1}{2}, 0\right)$, arXiv:0711.4624 [math.QA]

[18] X. Zhang, S. Tan, H. Lian, Unitary modules for the twisted HeisenbergVirasoro algebra, arXiv:1201.1338 [math.RA]

[19] Y. Huang, K. Misra, Lie algebras, vertex operator algebras and their applications, American Mathematical Society, Portland, 2007. 